\title{
El Aragoniense Medio y Superior en el Suroeste de Madrid: Los nuevos yacimientos de Micromamíferos del Mioceno Medio de Villaviciosa de Odón y Leganés
}

\author{
The Middle and Upper Aragonian in the Southwest of Madrid: The new \\ Micromammal sites of the Middle Miocene of Villaviciosa de Odón and \\ Leganés
}

\author{
C. Sesé 1 , J.C. Jiménez Rodrigo² \\ ${ }^{1}$ Museo Nacional de Ciencias Naturales, CSIC, Dpto. de Paleobiología, C/ José Gutiérrez Abascal, 2, 28006 Madrid, España. \\ Email: c.sese@mncn.csic.es \\ ${ }^{2}$ C/Bancaleros, 8, 45600 Talavera de la Reina, Toledo, España
}

\section{RESUMEN}

\begin{abstract}
En este trabajo se dan a conocer dos nuevos yacimientos de la zona suroeste de Madrid, Villaviciosa de Odón y Leganés, que han proporcionado dos ricas asociaciones de micromamíferos de edades diferentes. La asociación de micromamíferos del yacimiento de Villaviciosa de Odón determinada en este trabajo está compuesta por los siguientes taxones: Lagomorpha: Lagopsis peñai; Erinaceomorpha: Galerix cf. exilis; Soricomorpha: Miosorex cf. grivensis; Rodentia: Atlantoxerus sp., Microdyromys sp., Armantomys tricristatus, Megacricetodon collongensis y Democricetodon sp. Por el estadio evolutivo de Megacricetodon collongensis esta asociación se incluiría en la biozona local E de Daams \& Freudenthal (1988a) del final del Aragoniense Medio, correlacionable con el final de la unidad MN 5 de Mein (1975). La asociación del yacimiento de Leganés se compone de los siguientes taxones: Lagomorpha: Lagopsis verus; Erinaceomorpha: Galerix cf. exilis; Rodentia: Heteroxerus cf. rubricati, Armantomys sp., Megacricetodon collongensis-crusafonti y cf. Democricetodon sp. Por el estadio evolutivo de Megacricetodon collongensis-crusafonti se situaría en el Aragoniense Superior en la biozona local G, de Daams et al. (1999 a y b) que se correlaciona con la unidad MN 6 de Mein (1975). Es el primer yacimiento que se conoce en Madrid de esta edad. Las asociaciones de micromamíferos de Villaviciosa de Odón y Leganés sugieren un medio fundamentalmente abierto, con predominio de la vegetación dura de tipo estepario, y un clima seco y relativamente cálido.
\end{abstract}

Palabras clave: Micromamíferos; Mioceno Medio; Aragoniense Medio y Superior; Madrid; España

\section{ABSTRACT}

This paper discloses two new sites in the southwestern area of Madrid, Villaviciosa de Odón and Leganés, which have provided two rich micromammals associations of different ages. The micromammal association of the Villaviciosa de Odón site determined in this work is composed by the following taxa: Lagomorpha: Lagopsis peñai; Erinaceomorpha: Galerix cf. exilis; Soricomorpha: Miosorex cf. grivensis; Rodentia: Atlantoxerus sp., Microdyromys sp., Armantomys tricristatus, Megacricetodon collongensis and Democricetodon sp. This association, mainly by the

Recibido el 11 de noviembre de 2013 / Aceptado el 4 de marzo de 2014 / Publicado online el 27 de junio de 2014

Citation / Cómo citar este artículo: C. Sesé \& J.C. Jiménez Rodrigo (2014). El Aragoniense Medio y Superior en el Suroeste de Madrid: Los nuevos yacimientos de Micromamíferos del Mioceno Medio de Villaviciosa de Odón y Leganés. 70(1): e006. http://dx.doi. org/10.3989/egeol.41589.303.

Copyright: () 2014 CSIC. This is an open-access article distributed under the terms of the Creative Commons Attribution-Non Commercial (by-nc) Spain 3.0 License. 
evolutionary stage of Megacricetodon collongensis, corresponds to the E local biozone after Daams \& Freudenthal (1988a) from the end of the Middle Aragonian which is correlated with the end of the MN 5 Unit of Mein (1975). The micromammal association of the Leganés site consists of the following taxa: Lagomorpha: Lagopsis verus; Erinaceomorpha: Galerix cf. exilis; Rodentia: Heteroxerus cf. rubricati, Armantomys sp., Megacricetodon collongensis-crusafonti and cf. Democricetodon sp. Mainly by the evolutionary stage of Megacricetodon collongensiscrusafonti this association corresponds to the $G_{1}$ local biozone after Daams et al. (1999 a y b) which is correlated with the MN 6 Unit of Mein (1975). It is the only site known in Madrid of this age. Both micromammals associations suggest an open environment dominated by hard vegetation of steppe kind, and a dry and relatively warm climate.

Keywords: Micromammals; Middle Miocene; Middle and Upper Aragonian; Madrid; Spain

\section{Introducción y antecedentes}

El gran potencial paleontológico de la Comunidad de Madrid es conocido desde finales del siglo XIX por el hallazgo de numerosos yacimientos miocenos con restos de macromamíferos. Desde finales de los años 70 del siglo XX, los depósitos neógenos de la Comunidad de Madrid han librado una gran cantidad de yacimientos con restos también de micromamíferos. El primer yacimiento excavado en el que se aplicaron las técnicas de lavado-tamizado de sedimento para la obtención de microvertebrados fue el de Moratines, cuyos resultados se dieron a conocer en Alberdi et al. (1980) de forma preliminar y en Alberdi et al. (1981) con el estudio detallado de su fauna de mamíferos. Las diferentes obras de construcción y reparación de infraestructuras en la Comunidad de Madrid, que iban sacando a la luz yacimientos como el mencionado de Moratines y otros, motivaron el inicio de diversos proyectos de investigación paleontológica. Se comenzó así la prospección sistemática en el área metropolitana y la zona este de la Comunidad de Madrid (triángulo Cerro del Viso - Cerro de Almodóvar - Cerro de los Guardias), que llevaron a cabo el MNCN (CSIC), el IGME y la Facultad de Ciencias Geológicas de la UCM en estrecha colaboración. Fruto del desarrollo de estos proyectos son: la monografía coordinada por Alberdi (1985) en la que se recogen los datos sobre los hallazgos paleontológicos de mamíferos (Sesé et al., 1985; Alberdi et al., 1985), y, posteriormente, el trabajo sobre micromamíferos de López Martínez et al. (1987). Todas estas publicaciones, que dieron a conocer las faunas de mamíferos de doce nuevos yacimientos, supusieron un gran avance, sentando las bases para el conocimiento geológico, paleontológico y bioestratigráfico de los depósitos del Neógeno de la Comunidad de Madrid.

La promulgación en 1998 de la Ley de Patrimonio Histórico de la Comunidad de Madrid, abrió el camino y favoreció el desarrollo y protección de su rico patrimonio paleontológico. Resultado de ello fue el hallazgo de nuevos yacimientos de mamíferos y la excavación de los yacimientos clásicos, cuyos resultados se recogen en la monografía coordinada y editada por Morales et al. (2000a). En ella cabe mencionar: los trabajos de síntesis sobre los macroy micromamíferos de los diversos yacimientos miocenos de la Comunidad de Madrid conocidos hasta entonces (Peláez-Campomanes et al., 2000; Soria et al., 2000); también sobre el yacimiento vallesiense del Cerro de los Batallones (Morales et al., 2000b), yacimiento del que Morales et al. (1992) habían realizado un estudio preliminar y que posteriormente sería objeto de varios estudios detallados por Morales et al. (2004 y 2008); y sobre los micromamíferos del Aragoniense Medio de Somosaguas (López Martínez et al., 2000a), yacimiento este último del que posteriormente Luis \& Hernando (2000) estudiaron los micromamíferos, y López Martínez et al. (2000b) y Hernández Fernández et al. (2006) realizaron sendas síntesis. Posteriormente se han hallado otros yacimientos miocenos con micromamíferos en la Comunidad de Madrid: los yacimientos aragonienses de: Mesegar 1 y 2, Móstoles y Villaluenga-Cerro del Águila (López Olmedo et al., 2003 y 2004); Ramal Parla, Ramal Fuenlabrada y EDAR Culebro, con restos de plantas fósiles, y uno de ellos (Ramal Parla P.K. 1.250), también con micromamíferos (Fernández Marrón et al., 2002 y 2004); Nogales (Herráez et al., 2006); Casa Montero (López Guerrero et al., 2007); El Cañaveral (Hernández-Ballarín et al. (2010) y Húmera (Cárdaba et al., 2013). Peláez-Campomanes et al. (2003) y Hernández-Ballarín et al. (2011), en sus respectivos trabajos, reunieron la mayor parte de esta información y realizaron sendas síntesis biostratigráficas de los depósitos neógenos de la Comunidad de Madrid.

El hallazgo y estudio en este trabajo de los dos nuevos yacimientos con micromamíferos del 
Aragoniense Medio y Aragoniense Superior, permite profundizar en el conocimiento evolutivo y bioestratigráfico de las faunas de mamíferos de la Comunidad de Madrid, y en especial para las correspondientes al Aragoniense Superior, faunas de las que, en general, había hasta el momento pocos datos, y ninguno de la biozona local $\mathrm{G}_{1}$ en la Cuenca de Madrid.

\section{Geología}

\section{Localización geológica general}

Desde un punto de vista geológico, los yacimientos aquí estudiados se ubican en la Cuenca del Tajo y dentro a su vez de la subcuenca de Madrid, que se corresponde con una amplia depresión de origen tectónico, de más de $15.000 \mathrm{~km}^{2}$ de extensión, con escasos relieves dominados por amplias planicies denominadas páramos.

La Cuenca de Madrid tiene forma triangular, enmarcada por relieves montañosos: el Sistema Central (NE a SO), la Sierra de Altomira como límite oriental y los Montes de Toledo como margen meridional. Esta cuenca está rellena casi en su totalidad por sedimentos Paleógenos y Neógenos que descansan sobre un sustrato de materiales Cretácicos, apoyados a su vez en el zócalo cristalino, verdadero soporte de la cubeta (Junco \& Calvo, 1983). La sedimentación continental de la Cuenca de Madrid es de carácter endorreico y corresponde a depósitos detríticos (abanicos aluviales) procedentes del Sistema Central y de los Montes de Toledo, que, después de una facies intermedia de transición, dan paso, hacia el centro de la cuenca, a sedimentos químicos lacustres carbonatados y evaporíticos.

Los yacimientos aquí estudiados se sitúan en el sector central de la Cuenca de Madrid, dentro de los términos medios de la sedimentación Miocena, en la llamada facies de borde (Vegas et al., 1975), caracterizada litológicamente por arcosas y arcillas arenosas depositadas por un sistema de abanicos aluviales de composición eminentemente arcósica procedentes del Sistema Central.

\section{Localización espacio-temporal}

El yacimiento de Villaviciosa de Odón se encontró en el año 2000 en la localidad del mismo nombre, en una urbanización en la que se proyectaba construir casas en el Área de Planeamiento Integrado, denominado "Sector I" La Tenería.

El yacimiento de Leganés se descubrió en el año 2001 en el marco de la prospección de las obras de la EDAR (Estación Depuradora de Aguas Residuales Urbanas) del arroyo Culebro, y dentro de los ramales o emisarios que conducen a dicha depuradora que, numerados por orden de finalización son: Ramal Parla, Ramal Leganés Norte, Ramal Leganés Sur, Ramal Tramo Común, Ramal Fuenlabrada y obras en la EDAR arroyo Culebro. El yacimiento objeto del presente trabajo está situado concretamente en los terrenos pertenecientes al llamado Ramal o Emisario de Leganés Sur, que consistió en una canalización realizada para comunicarse con la EDAR de la cuenca alta del arroyo Culebro. La apertura de los frentes de estos colectores permitieron que afloraran secciones muy continuas, aunque de escasa profundidad (ver columna estratigráfica en la Fig. 2), de cuyos niveles se extrajeron restos de plantas y vertebrados fósiles (Fernández Marrón et al., 2002 y 2004).

El yacimiento objeto del presente trabajo, llamado Leganés para abreviar, corresponde al Ramal Depuradora Leganés Sur PK 500 de los ramales anteriormente mencionados, que se sitúa a una cota de 649 metros sobre el nivel del mar, dentro del término municipal de Leganés y cercano a la estación de La Serna en Fuenlabrada. Con respecto al yacimiento de Villaviciosa de Odón está situado a unos $12 \mathrm{~km}$ al sur.

Durante el Terciario, el área donde se ubican los yacimientos aquí estudiados sufre la fracturación del zócalo antiguo Precámbrico-Paleozoico, a consecuencia de una de las grandes orogenias que afectaron a la Comunidad de Madrid: la orogenia Alpina (que dio origen a los Pirineos, Alpes, Himalaya, etc.).

Durante esta orogenia se produjo un levantamiento y hundimiento de bloques, generándose la gran Depresión o Cuenca del Tajo, que empezó a rellenarse mediante la acción de cursos fluviales con procedencia de dos áreas fuente: una septentrional del Sistema Central y otra meridional de los Montes de Toledo. Estos sistemas aluviales, según perdían competencia, organizaron los materiales por tamaños granulométricos, apareciendo los materiales gruesos en los bordes de la cuenca, más próximos a la sierra, y los finos hacia el centro de la cuenca. Así, de depósitos detríticos: conglomerados, arcosas 


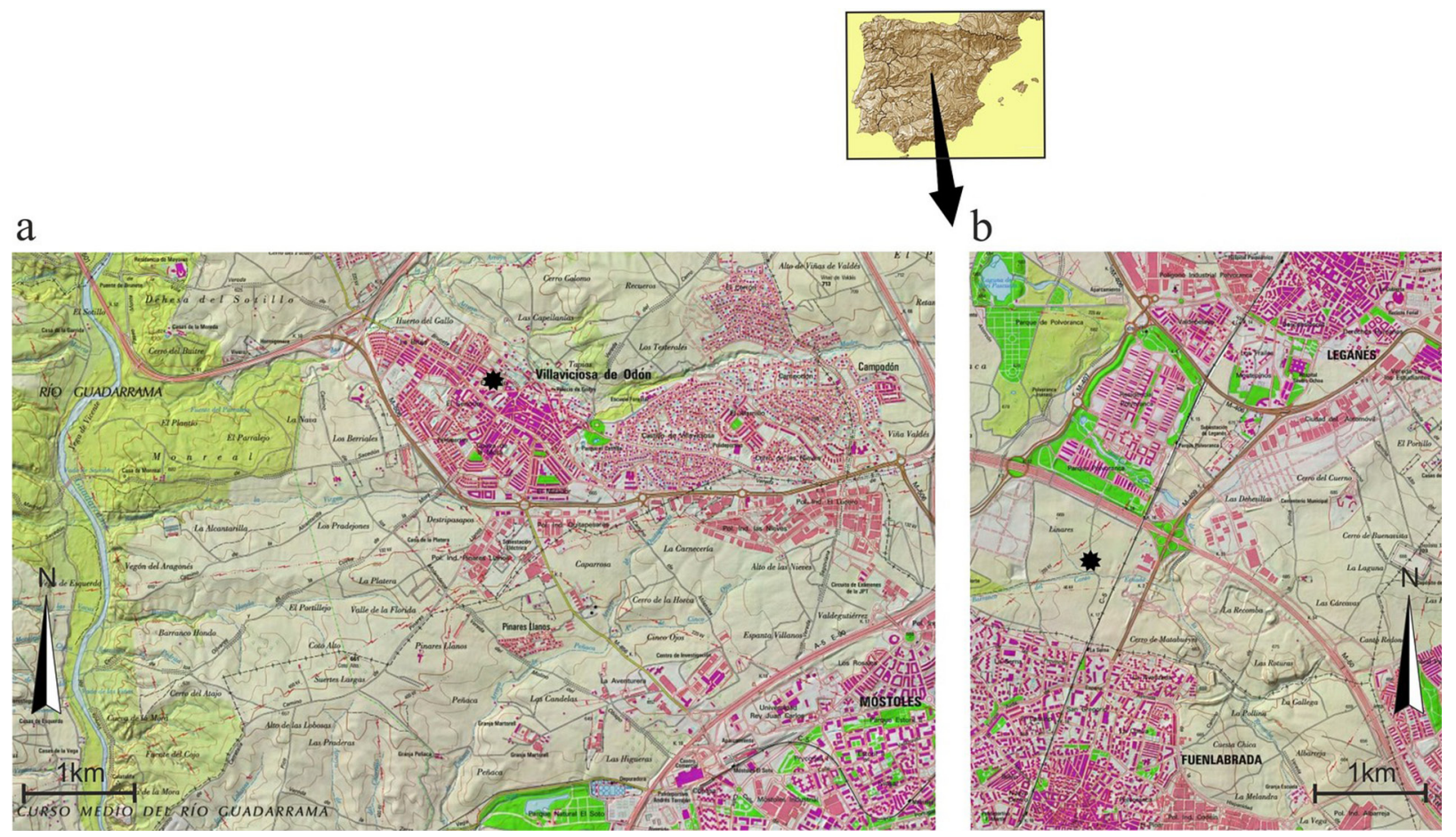

Fig. 1.-Mapa de situación de los yacimientos. Con el signo del asterisco $\left(^{*}\right)$ se señala la ubicación de los yacimientos: a) Villaviciosa de Odón, y b) Leganés.

y arcillas, se pasa a una facies intermedia de transición constituida por arenas micáceas y lutitas que dan paso hacia el centro de la cuenca a sedimentos químicos constituidos por carbonatos de lagos someros y evaporitas (Hoyos et al., 1985).

Los nuevos yacimientos aparecen dentro de los depósitos detríticos de arcosas y arcillas, situándose el yacimiento de Leganés muy cerca de la zona intermedia de transición (o depósitos de transición) que constituye una banda bastante continua, entre los sistemas de abanicos aluviales (donde se encuentran estos dos yacimientos) compuestos de litologías eminentemente arcósicas y con procedencia del Sistema Central, y las facies lacustres del centro de la cuenca (Calvo et al., 1984 y 1989; Vegas et al., 1975). Estos autores (o.c.) interpretan el medio como facies más distales de los abanicos aluviales y cercanos a las facies lacustres.

El yacimiento de Villaviciosa de Odón aparece muy cerca del contacto entre las arcosas y fangos arcósicos (17, según la numeración de los autores de la hoja de Majadahonda) y fangos arcósicos y arcosas (18, según la numeración de los autores de dicha hoja) (Portero García \& Pérez González, 1990), dentro de las primeras, y cerca de donde dichos autores de la hoja de Majadahonda, en la cota 615 metros, hallaron dos mandíbulas inferiores de Bunolistriodon lockarti (Urbanización el Bosque) que atribuyeron al Aragoniense Inferior; no obstante, hay que señalar que en la Cuenca de Madrid esta especie se registra durante el Aragoniense Medio (biozonas locales Dc, Dd y E), desapareciendo al final de dicho periodo, según Soria et al. (2000), por lo que su amplio registro biostratigráfico no permite asegurar su edad del Aragoniense Inferior. Portero García \& Pérez González (1990) interpretan el medio como facies más distales o laterales de los grandes sistemas deposicionales de abanicos aluviales que contienen una mayor proporción de finos de llanura de inundación.

\section{Descripción de las columnas estratigráficas}

Las columnas estratigráficas se han levantado en los puntos donde aparecen los yacimientos de fósiles. Las capas se encuentran horizontales. 


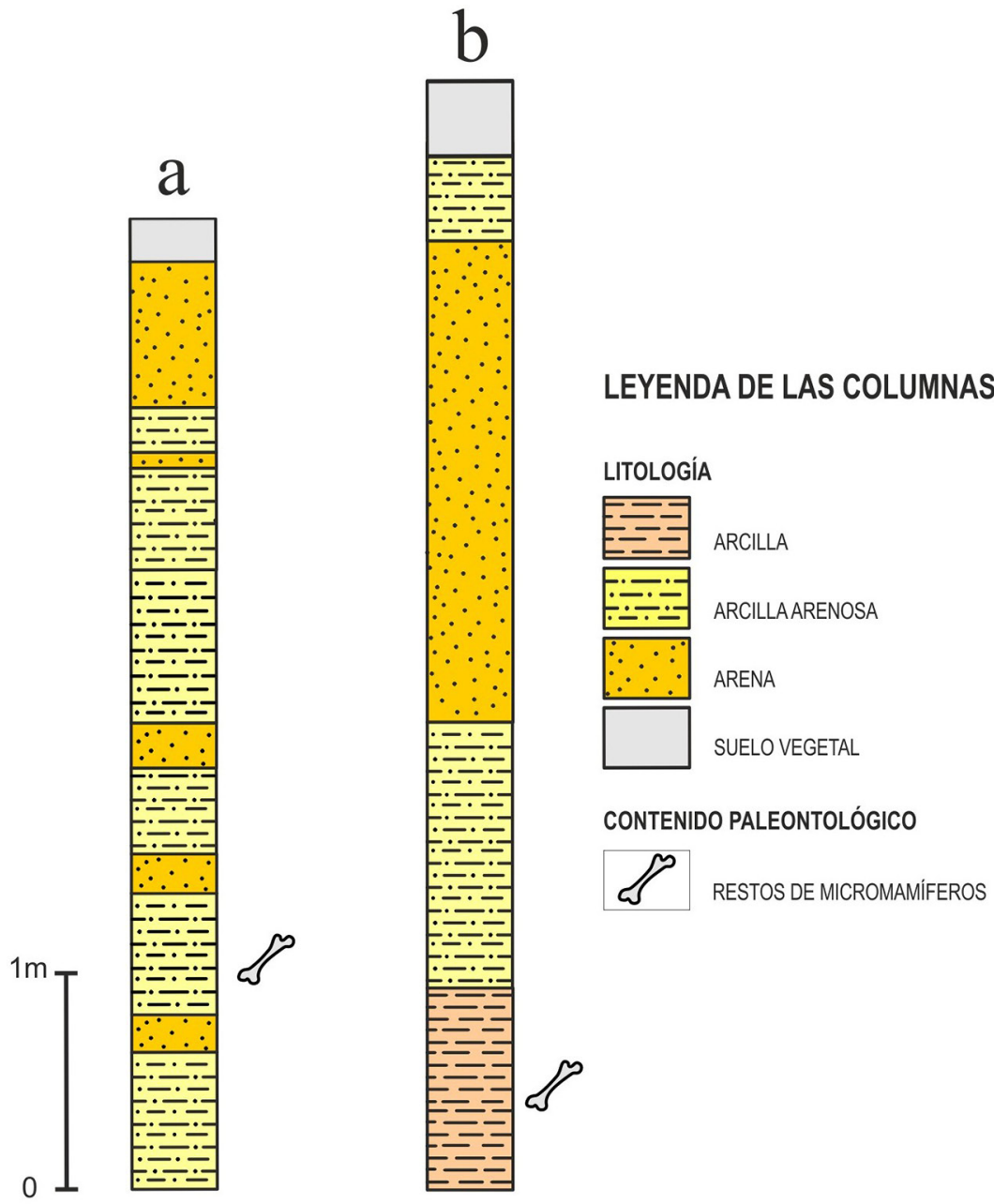

Fig. 2.-Estratigrafía de los yacimientos de Villaviciosa de Odón y Leganés. Columnas litológicas de los yacimientos de: a) Villaviciosa de Odón, y b) Leganés.

El yacimiento de Villaviciosa de Odón se encuentra en una cota de 630 metros sobre el nivel del mar, hoja número 558, Majadahonda, en el término municipal de Villaviciosa de Odón y al noroeste de dicho municipio. Sus coordenadas geográficas son: Longitud: $3^{\circ} 54^{\prime} 36^{\prime \prime}$ Oeste; Latitud: $40^{\circ} 21^{\prime} 49^{\prime \prime}$ Norte.

La estratigrafía de detalle en un corte del terreno afectado por las obras de la urbanización muestra las siguientes sucesiones de niveles de muro a techo:

- 0,58 metros de arcilla arenosa.

- 0,20 metros de arcosa arcillosa fina color pardorojizo con moteados de manganeso.
- 1,10 metros de arcilla parda arenosa con presencia de nódulos y con numerosas placas de tortugas y fragmentos óseos, además de restos de microvertebrados. Presenta un canal de unos $2 \mathrm{~m}$ de anchura y $0,20 \mathrm{~m}$ de espesor máximo que se encuentra relleno con arena gruesa-media.

- 0,20 metros de arena fina arcillosa de color claro, pasando hacia el este de la columna a un pequeño canal de $0,57 \mathrm{~m}$ de espesor con pasadas de arena más gruesa y laminación cruzada en surco.

- 0,70 metros de arcilla arenosa de color pardo rojizo con nódulos.

- $\quad$,70 metros de arcilla arenosa color verde muy húmeda y carbonatada con pasada de arena de $0,05 \mathrm{~m}$ que forma un canal hacia el oeste. 
- $\quad$,70 metros de arenas de color pardo de origen coluvionar cuaternarias.

- 0,20 metros de suelo vegetal oscuro.

El yacimiento de Leganés (Ramal Depuradora Leganés Sur PK 500) se sitúa a una cota de 650 metros sobre el nivel del mar, en la hoja 19-23 (582) Getafe 1998, dentro del término municipal de Leganés, al suroeste de dicha localidad y en las proximidades de Fuenlabrada. Sus coordenadas geográficas son: Longitud: $3^{\circ} 47^{\prime} 33^{\prime \prime}$ Oeste; Latitud: $40^{\circ} 18^{\prime} 13^{\prime \prime}$ Norte. Está a unos $12 \mathrm{~km}$ al sur del yacimiento de Villaviciosa de Odón.

La estratigrafía de detalle muestra las siguientes sucesiones de niveles de muro a techo de la zanja y pre-zanja en su cara norte:

- 0,91 metros de arcilla de color rojizo con un moteado por tinción de manganeso y con ligera laminación por micas. Presenta numerosos restos de huesos pequeños de microvertebrados fósiles. Muy compactas.

- 1,21 metros de arcilla arenosa fina de color verde. Erosionado a techo por el canal de arenas.

- 2,25 metros de arena de tamaño medio-grueso de color amarillento con algunos cantos de cuarcita y cuarzo dispersos. Representa un canal arenoso con base canalizada.

- 0,46 metros de arcilla verde arenosa con manchas de carbonatos de origen edáfico.

- $\quad 0,35$ metros de suelo vegetal, de color gris y con aspecto prismático.

\section{Material y métodos}

Se lavaron con agua $175 \mathrm{~kg}$ de sedimento del yacimiento de Villaviciosa de Odón y $410 \mathrm{~kg}$ del yacimiento de Leganés, haciéndolo pasar por una serie de tamices superpuestos de distintos tamaños de luz de malla de $2 \mathrm{~mm}, 1,5 \mathrm{~mm}, 1 \mathrm{~mm}$ y $0,5 \mathrm{~mm}$.

Los dientes se han medido con una lupa que lleva incorporado un micrómetro de $0,025 \mathrm{~mm}$ de precisión. Las medidas, tomadas en la superficie oclusal de los dientes orientados en su correspondiente posición anatómica, se expresan en milímetros $(\mathrm{mm}) \mathrm{y}$ corresponden a la longitud (L) y anchura (A) máximas. La nomenclatura utilizada para los dientes es según López Martínez (1989) para los lagomorfos, Daams \& Freudenthal (1988b) para los cricétidos y
Sesé (1980) para el resto de los grupos. Los dibujos se han realizado utilizando una cámara clara acoplada a un microscopio Nikon SMZ-IO con 40 aumentos. Para comparar la abundancia relativa de los taxones se ha utilizado el NR (Número de Restos) y el NMI (Número Mínimo de Individuos) calculado sobre la pieza dentaria taxonómicamente identificable más abundante, teniendo en cuenta el número máximo de piezas derechas o izquierdas. Para evitar la sobrerrepresentación que puede suponer la utilización del NR en algunos grupos, teniendo en cuenta que hay algunos como los lagomorfos que tienen más dientes en general que los roedores, el porcentaje (\%) de abundancia relativa se ha calculado sobre el NMI. Las abreviaturas utilizadas son: MNCN (Museo Nacional de Ciencias Naturales), CSIC (Consejo Superior de Investigaciones Científicas), IGME (Instituto Geológico y Minero de España), UCM (Universidad Complutense de Madrid), N (Número de dientes), MÍN. (Mínima), MED. (Media), MÁX. (Máxima), D/P/M (Diente de leche/Premolar/Molar con el número que indica la posición del diente, en subíndice para los inferiores y en superíndice para los superiores). Cuando se mencionan en el texto los yacimientos madrileños refiriéndolos a Madrid, se hace para abreviar, ya que se trata de los de la Comunidad de Madrid en general, tanto si están situados en Madrid capital como si están en alguno de los pueblos de dicha comunidad. El material está depositado en las colecciones del MNCN.

\section{Paleontología}

\section{Yacimiento de Villaviciosa de Odón}

Orden Lagomorpha BRANDT, 1855

Familia Ochotonidae THOMAS, 1897

Lagopsis peñai (ROYO, 1928)

(Fig. 3: 1 y 2)

Material: $4 \mathrm{P}_{3}$ der.; $2 \mathrm{P}_{3}$ izq.; 3 frs. $\mathrm{P}_{3}$ izq.; $3 \mathrm{P} / \mathrm{M}$ inf.; $2 \mathrm{P}^{2}$ der.; 1 fr. $\mathrm{P}^{2}$ der.; $13 \mathrm{P} / \mathrm{M}$ sup.; $\mathrm{NR}=28$; NMI $=5$

Medidas:

\begin{tabular}{|c|c|c|c|c|c|c|c|c|}
\hline \multirow[b]{2}{*}{ DIENTE } & \multirow[b]{2}{*}{$\mathrm{N}$} & \multicolumn{3}{|c|}{ LONGITUD } & & \multicolumn{3}{|c|}{ ANCHURA } \\
\hline & & MíN. & MED. & MÁX. & $\mathrm{N}$ & MÍN. & MED. & MÁX. \\
\hline$P_{3}$ & 6 & 1,74 & 1,84 & 1,97 & 5 & 1,72 & 1,75 & 1,81 \\
\hline$P^{2}$ & 1 & - & 0,92 & - & 1 & - & 1,92 & - \\
\hline
\end{tabular}



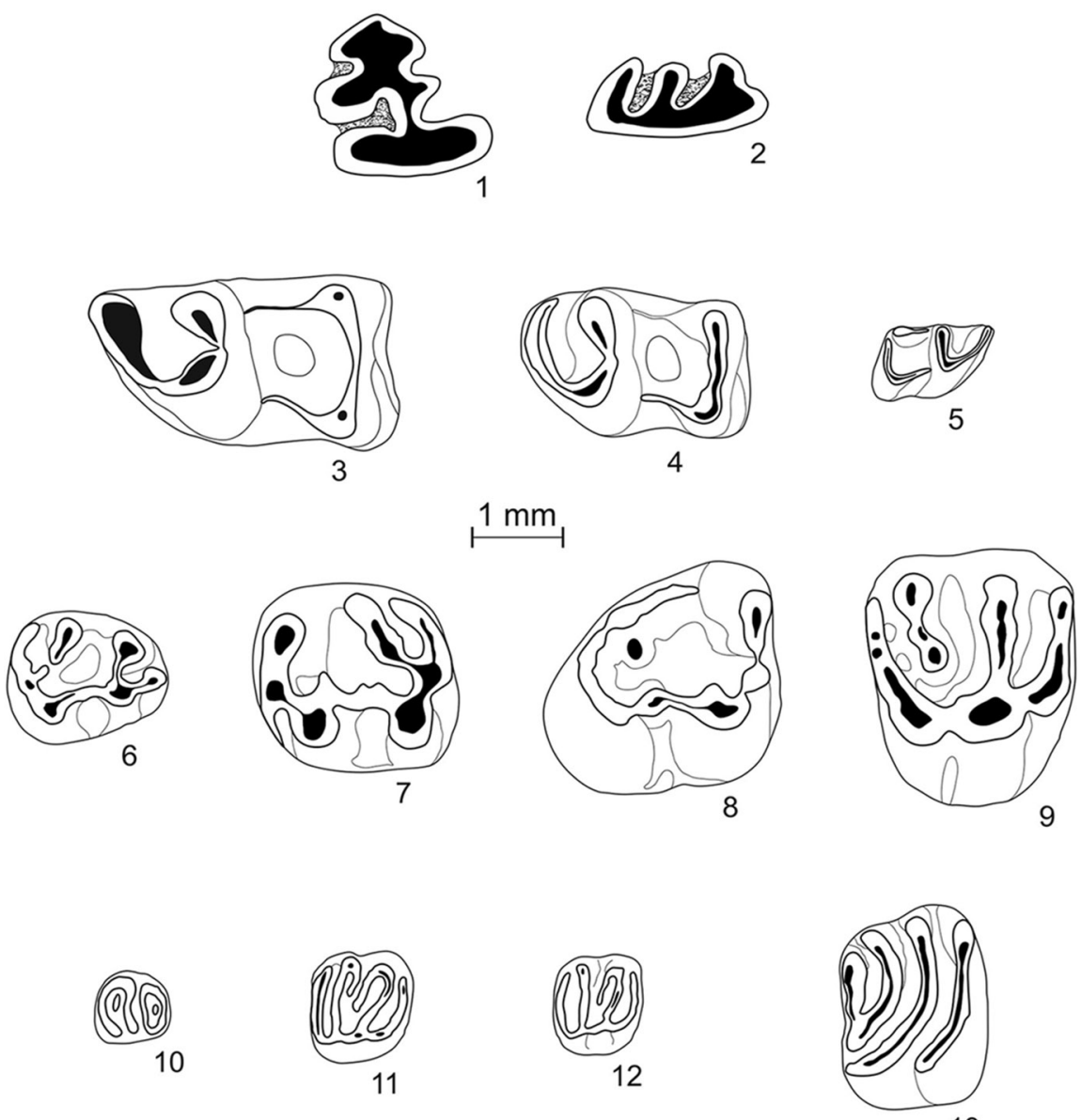

13
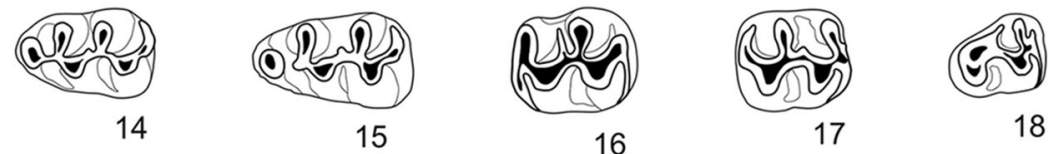

16

17

18

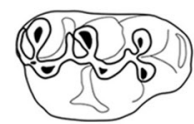

19

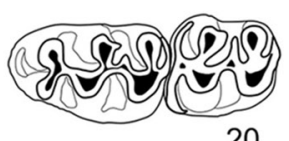

20

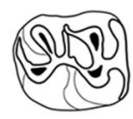

21

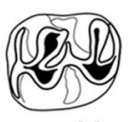

22

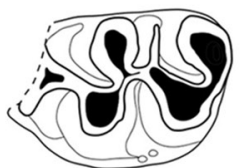

23

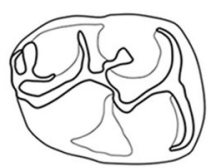

24

Fig. 3.-Dibujos de los dientes de micromamíferos en vista oclusal del yacimiento de Villaviciosa de Odón: Lagopsis peñai: 1: $P_{3}$ der. (VCT 5); 2: $\mathrm{P}^{2}$ der. (VCT 9); Galerix cf. exilis: 3: $\mathrm{M}_{1}$ izq. (VCT 25); 4: $\mathrm{M}_{2}$ izq. (VCT 26); Miosorex cf. grivensis: 5 : $\mathrm{M}_{1}$ der. (VCT 29); Atlantoxerus sp.: 6: $\mathrm{P}_{4}$ der. (VCT 63); 7: $\mathrm{M}_{1-2}$ izq. (VCT 90); 8: $\mathrm{M}_{3}$ der. (VCT 89); 9: $\mathrm{M}^{1-2}$ der. (VCT 88); Microdyromys sp.: 10 : 1 $\mathrm{P}_{4}$ der. (VCT 116); 11: $\mathrm{M}^{1-2}$ der. (VCT 118); 12: $\mathrm{M}^{1-2}$ der. (VCT 117); Armantomys tricristatus: 13: $\mathrm{M}^{1-2}$ der. (VCT 148); Megacricetodon collongensis: 14: $M_{1}$ izq. (VCT 104); 15: $M_{1}$ izq. (VCT 125); 16: $M_{2}$ izq. (VCT 70); 17: $M_{2}$ der. (VCT 121); 18: $M_{3}$ der. (VCT 72); 19: $M^{1}$ der. (VCT 127); 20: $\mathrm{M}^{1}-\mathrm{M}^{2}$ izq. (VCT 122-123); 21: $\mathrm{M}^{2}$ der. (VCT 68); 22: $\mathrm{M}^{2}$ izq. (VCT 67); Democricetodon sp.: 23: fr. $\mathrm{M}^{1}$ izq. (VCT 65); 24: $\mathrm{M}^{2}$ der. (VCT 66). 
Descripción y discusión: La morfología del $\mathrm{P}_{3}$ es la característica de Lagopsis peñai: anterocónido reducido, de forma subtriangular, sin anterofléxido, y metacónido también reducido y de contorno redondeado (López Martínez, 1989). La talla del material es similar a la de las poblaciones de dicha especie de los yacimientos de Las Planas 4 B (López Martínez, 1989) y Somosaguas (Luis \& Hernando, 2000). Esta especie es muy común en los yacimientos españoles del Rambliense y Aragoniense Inferior y Medio (Sesé, 2006).

Orden Erinaceomorpha GREGORY, 1910

Familia Erinaceidae FISCHER, 1814

Galerix cf. exilis (BLAINVILLE, 1840)

(Fig. 3: 3 y 4)

Material: $1 \mathrm{P}_{4}$ izq.; $1 \mathrm{M}_{1}$ izq.; $1 \mathrm{M}_{2}$ izq.; 2 frs. $\mathrm{M}_{1-2}$ der.; $1 \mathrm{P}^{4}$ der.; 1 fr. $\mathrm{M}^{1}$ izq.; $1 \mathrm{M}^{3}$ der.; $\mathrm{NR}=8$; $\mathrm{NMI}=2$

Medidas: $1 \mathrm{M}_{1}(\mathrm{~L}=3,26 ; \mathrm{A}=1,99) ; 1 \mathrm{M}_{2}(\mathrm{~L}=2,47$; $\mathrm{A}=1,66) ; 1 \mathrm{P}^{4}(\mathrm{~L}=2,61 ; \mathrm{A}=2,89)$

Descripción y discusión: Comparando este material con la abundante población de esta especie del yacimiento del Aragoniense Superior de Escobosa de Calatañazor (Sesé, 1980), muestra una completa similitud morfológica y biométrica. Sin embargo, aunque el $\mathrm{M}_{2}$ y el $\mathrm{P}^{4}$ entran en la variabilidad de la talla de las poblaciones de dicha especie del Mioceno de la Cuenca de Calatayud-Teruel según Jong (1988), el $M_{1}$ es de mayor talla, y el $M_{1}$ y $M_{2}$ son también de mayor talla que los del yacimiento aragoniense de Somosaguas (Luis \& Hernando, 2000). Con respecto a la especie rambliense Galerix remmerti HOEK OSTENDE, 2003, la talla del $\mathrm{M}_{2}$ está dentro de la variabilidad de las poblaciones de dicha especie de Daroca-Calamocha, pero el $\mathrm{M}_{1}$ y el $\mathrm{P}^{4}$ son de mayor talla según los datos de Hoek Ostende (2003). Al faltar en el material piezas dentarias que presenten caracteres con valor diagnóstico para la especie, realizamos su atribución con reservas, razón por la que la denominamos Galerix cf. exilis. Galerix exilis es una especie muy común en los yacimientos peninsulares españoles del Aragoniense (Gibert, 1975; Jong, 1988; Hoek Ostende \& Furió, 2005). En el Mioceno Medio de Madrid el género Galerix se registra en muchos yacimientos, bien citado como Galerix sp. o Galerix exilis. El último registro conocido del género Galerix en España es en la Cuenca de
Calatayud-Teruel al comienzo del Vallesiense (Jong, 1988; Hoek Ostende, 2001).

Orden Soricomorpha GREGORY, 1910

Familia Soricidae FISCHER, 1814

Miosorex cf. grivensis (DEPERET, 1892)

(Fig. 3: 5)

Materialy Medidas: 1 fr. $\mathrm{M}_{1}$ der.; $1 \mathrm{M}_{1}$ izq. ( $\mathrm{L}=1,32$; $\mathrm{A}=0,84) ; 1$ fr. $\mathrm{M}^{1-2}$ lado indet.; $\mathrm{NR}=3$; $\mathrm{NMI}=1$

Descripción y discusión: El $\mathrm{M}_{1}$ que está completo es similar en morfología y talla al de Miosorex grivensis lopezae de Escobosa de Calatañazor (Sesé, 1980) y a las poblaciones aragonienses de Miosorex grivensis de la Cuenca de Calatayud-Teruel (Jong, 1988). Sin embargo, dada la escasez y fragmentariedad del material, y en ausencia de piezas más características, se determina como Miosorex cf. grivensis por su afinidad con dicha especie. En el yacimiento del Aragoniense Medio de Madrid de Somosaguas se cita cf. Miosorex grivensis (Luis \& Hernando, 2000; Hernández Fernández et al., 2006).

Orden Rodentia BOWDICH, 1821

Familia Sciuridae FISCHER DE WALDHEIM, 1817

\section{Atlantoxerus sp.}

(Fig. 3: 6-9)

Material: $1 \mathrm{P}_{4}$ der.; $1 \mathrm{M}_{1-2}$ izq.; 1 fr. $\mathrm{M}_{1-2}$ izq.; $2 \mathrm{M}_{3}$ der.; $1 \mathrm{D}^{4}$ izq.; $1 \mathrm{P}^{4}$ izq.; $2 \mathrm{M}^{1-2}$ der. ; 1 fr. $\mathrm{M}^{1-2}$ der.; 1 $\mathrm{M}^{3}$ izq.; $\mathrm{NR}=11 ; \mathrm{NMI}=2$

Medidas:

\begin{tabular}{lccccccccc}
\hline & & \multicolumn{3}{c}{ LONGITUD } & & \multicolumn{3}{c}{ ANCHURA } \\
\cline { 7 - 9 } DIENTE & $N$ & MÍN. & MED. & MÁX. & $N$ & MÍN. & MED. & MÁX. \\
\hline $\mathrm{P}_{4}$ & 1 & - & 1,71 & - & 1 & - & 1,46 & - \\
$\mathrm{M}_{1-2}$ & 1 & - & 2,18 & - & 1 & - & 2,14 & - \\
$\mathrm{M}_{3}$ & 2 & 2,27 & 2,45 & 2,63 & 2 & 2,32 & 2,32 & 2,33 \\
$\mathrm{P}^{4}$ & 1 & - & 2,07 & - & 1 & - & 2,22 & - \\
$\mathrm{M}^{1-2}$ & 2 & 2,06 & 2,16 & 2,26 & 2 & 2,53 & 2,55 & 2,58 \\
$\mathrm{M}^{3}$ & 1 & - & 2,21 & - & 1 & - & 2,24 & - \\
\hline
\end{tabular}

Descripción y discusión: Su morfología, con cúspides y crestas robustas, cónulos fuertes y corona hipsodonta, y su mayor talla, le diferencian claramente del género Heteroxerus y le asimilan al género Atlantoxerus según la diagnosis realizada por 
Cuenca Bescós (1988). Su talla es menor que la de Atlantoxerus blacki (BRUIJN, 1967) y mayor que la de Atlantoxerus idubedensis CUENCA BESCÓS, 1988 (Bruijn, 1967; Cuenca Bescós, 1988), dos especies del Aragoniense en España que esta última autora (o.c.) relaciona filogenéticamente. En Madrid se cita Atlantoxerus blacki en los yacimientos del Aragoniense Medio de Estación Imperial y Paseo de las Acacias, situados en la biozona local Dc (Soria et al., 2000; Peláez-Campomanes et al., 2003).

Familia Gliridae MUIRHEAD, 1819

\section{Microdyromys sp.}

(Fig. 3: 10-12)

Material: $1 \mathrm{P}_{4}$ der.; $1 \mathrm{P}^{4}$ der.; $2 \mathrm{M}^{1-2}$ der.; $\mathrm{NR}=4$; $\mathrm{NMI}=1$

Medidas:

\begin{tabular}{lcccccccccc}
\hline & & \multicolumn{3}{c}{ LONGITUD } & & \multicolumn{3}{c}{ ANCHURA } \\
\cline { 7 - 9 } DIENTE & $N$ & MÍN. & MED. & MÁX. & $N$ & MÍN. & MED. & MÁX. \\
\hline $\mathrm{P}_{4}$ & 1 & - & 0,81 & - & 1 & - & 0,70 & - \\
$\mathrm{P}^{4}$ & 1 & - & 0,56 & - & 1 & - & 0,75 & - \\
$\mathrm{M}^{1-2}$ & 2 & 0,92 & 1,01 & 1,10 & 2 & 1,06 & 1,15 & 1,23 \\
\hline
\end{tabular}

Descripción y discusión: Por la talla y la morfología simple de los dientes, sin crestas extra, presenta la mayor similitud con Microdyromys monspeliensis AGUILAR, 1977 y Microdyromys koenigswaldi de BRUIJN, 1966 (Daams, 1981; García Paredes, 2006), especies que son frecuentes en los yacimientos del Aragoniense de Madrid. Sin embargo, el material del yacimiento de Villaviciosa de Odón es muy escaso como para poder precisar la especie.

Armantomys tricristatus LÓPEZ MARTÍNEZ, 1977, in: LÓPEZ MARTÍNEZ, SESÉ \& SANZ, 1977

(Fig. 3: 13)

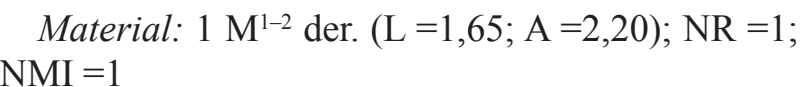

Descripción y discusión: Este molar presenta una morfología similar a la de Armantomys tricristatus del yacimiento del Aragoniense Superior de Escobosa de Calatañazor, donde fue definida la especie (López Martínez et al., 1977; Sesé, 1980): ausencia de centrolofos y crestas accesorias, corona dentaria muy hipsodonta, crestas muy inclinadas, oblicuas al eje longitudinal del diente, y posterolofo muy reducido; sin embargo, es de menor talla, no alcanzado los valores mínimos de ésta. Esto también ocurre en la población del yacimiento de Somosaguas que, con una morfología similar a dicha especie, tiene una talla menor que la de la población el yacimiento tipo de la especie, según Luis \& Hernando (2000). La talla del $\mathrm{M}^{2}$ del yacimiento de Villaviciosa a de Odón entra dentro de la variabilidad de la del yacimiento de Somosaguas, próxima a los valores medios.

Daams (1990) revisó el género Armantomys en España y distinguió dos líneas filogenéticas: una de talla pequeña a media: $A$. bijmai (LACOMBA \& MARTÍNEZ SALANOVA, 1988) - A. daamsi (VISSER, 1990) in: ÁLVAREZ SIERRA et al., 1990 - A. parsani DAAMS, 1990 - A. jasperi DAAMS, 1990, y otra de gran talla: A aragonensis BRUIJN, 1966 - A. tricristatus. Uno de los caracteres del $\mathrm{M}^{1-2}$ que según dicho autor (o.c.) separan estas dos últimas especies es que en $A$. tricristatus el posterolofo suele estar aislado o su extremo lingual se une al extremo lingual del metalofo. En el $\mathrm{M}^{1-2}$ de Villaviciosa de Odón esta unión se realiza cerca del extremo del metalofo; se trata de un ejemplar desgastado en el que en estadios más juveniles el posterolofo, muy posiblemente, estaría aislado, lo que le aproximaría todavía más a Armantomys tricristatus. Daams (1990) señala que, aunque $A$. aragonensis es de menor talla que $A$. tricristatus, los primeros representantes de ésta última son de talla intermedia, solapándose las de ambas. La talla del ejemplar de Villaviciosa de Odón se solapa con las de ambas especies según los datos de Daams (1990) y García Paredes (2006). Según Daams (1990), A. tricristatus comienza su registro en el Aragoniense Medio en la biozona $\mathrm{D}_{3}$ en yacimientos que posteriormente fueron situados en la biozona Dd de la biozonación propuesta por Daams et al. (1999 a y b). García Paredes (2006) realiza también un estudio morfológico y biométrico del género Armantomys y sitúa el límite entre ambas especies dentro de la biozona Dd. Según Luis \& Hernando (2000), en Madrid A. tricristatus aparece al comienzo de la biozona E. El material de Villaviciosa de Odón correspondería a un estadio inicial de $A$. tricristatus, especie que alcanzaría los estadios más progresivos de las tendencias 
evolutivas observadas en dicho linaje, así como una mayor talla, al final del Aragoniense, periodo al final del cual se extinguió el género (Daams, 1990; Sesé 2006). El género Armantomys es muy común en casi todos los yacimientos del Aragoniense Medio y Superior de Madrid.

Familia Cricetidae FISCHER, 1817 Megacricetodon collongensis (MEIN, 1958)

(Fig. 3: 14-22)

Material: $3 \mathrm{M}_{1}$ der.; $8 \mathrm{M}_{1}$ izq.; 2 frs. $\mathrm{M}_{1-2}$ izq.; 3 $\mathrm{M}_{2}$ der.; $4 \mathrm{M}_{2}$ izq.; $1 \mathrm{M}_{3}$ der.; 1 fr. maxilar sup. con $\mathrm{M}^{1}-\mathrm{M}^{2}$ izq.; $9 \mathrm{M}^{1}$ der.; $3 \mathrm{M}^{1}$ izq.; $3 \mathrm{M}^{2}$ der.; $3 \mathrm{M}^{2}$ izq.; $1 \mathrm{M}^{3}$ der.; $1 \mathrm{M}^{3}$ izq.; $\mathrm{NR}=43$; $\mathrm{NMI}=9$

Medidas:

\begin{tabular}{|c|c|c|c|c|c|c|c|c|}
\hline \multirow[b]{2}{*}{ DIENTE } & \multirow[b]{2}{*}{$\mathrm{N}$} & \multicolumn{3}{|c|}{ LONGITUD } & \multirow[b]{2}{*}{$\mathrm{N}$} & \multicolumn{3}{|c|}{ ANCHURA } \\
\hline & & MíN. & MED. & MÁX. & & MíN. & MED. & MÁX \\
\hline $\mathrm{M}_{1}$ & 8 & 1,43 & 1,49 & 1,58 & 8 & 0,87 & 0,92 & 0,98 \\
\hline $\mathrm{M}_{2}$ & 5 & 1,11 & 1,17 & 1,20 & 5 & 0,90 & 0,99 & 1,04 \\
\hline $\mathrm{M}_{3}$ & 1 & - & 0,99 & - & 1 & - & 0,82 & - \\
\hline $\mathrm{M}^{1}$ & 11 & 1,43 & 1,59 & 1,74 & 12 & 0,94 & 1,01 & 1,12 \\
\hline $\mathrm{M}^{2}$ & 4 & 1,12 & 1,15 & 1,18 & 4 & 0,93 & 0,98 & 1,02 \\
\hline $\mathrm{M}^{3}$ & 1 & - & 0,79 & - & 1 & - & 0,74 & - \\
\hline
\end{tabular}

Descripción: Se ha comparado morfológica y biométricamente el material de Villaviciosa de Odón con el de las diferentes especies del género Megacricetodon de la Cuenca de Calatayud-Teruel descritas por Daams \& Freudenthal (1988b), utilizando los morfotipos que proponen para los diferentes caracteres de los molares:

$\mathrm{M}_{1}$ : De 11 ejemplares, 10 tienen el anterocónido simple con forma redondeada o alargada (morfotipo 1) y solo uno tiene un anterocónido ligeramente dividido (morfotipo 2). En 2 ejemplares el mesolófido es medianamente largo, alcanzando la mitad del mesoseno (morfotipo 2), en 7 es corto (morfotipo 3 ) y en 2 no existe (morfotipo 4).

$\mathrm{M}_{2}$ : De 7 especímenes, en 4 hay un corto anterolófido lingual (morfotipo 2) y en 3 no existe (morfotipo 3 ). El mesolófido es medianamente largo alcanzado más o menos la mitad del mesosinúsido (morfotipo 2) en 3 ejemplares, es corto o apenas esbozado como una protuberancia (morfotipo 3 ) en 3 , y no existe (morfotipo 4) en uno.
$\mathrm{M}^{1}$ : De 14 ejemplares, en uno el anterocono está ligeramente dividido pero el surco no es muy profundo (morfotipo 2), en 9 está bien dividido por un surco profundo (morfotipo 3 ) y en 4 está dividido por un surco profundo y hay un pequeño cíngulo basal en la parte anterior frente al anterocono (morfotipo 4). Ningún ejemplar presenta una protuberancia labial en el anterolófulo. No existe ectolofo (morfotipo 1) en 3 ejemplares, en 7 es corto (morfotipo 2) y en 4 está bien desarrollado (morfotipo 3). El mesolofo es largo alcanzando el borde labial del diente (morfotipo 1) en un ejemplar, medianamente largo alcanzando la mitad del mesoseno (morfotipo 2) en 8 y es corto (morfotipo 3 ) en 5 . Solo en 2 ejemplares el ectolofo conecta con el mesolofo. Todos los ejemplares presentan posterolofo: en 2 el posterolofo es largo, delimitando un amplio posteroseno (morfotipo 1), en 6 es corto, delimitando un pequeño posteroseno (morfotipo 2) y en 6 es muy corto obliterando el posteroseno (morfotipo 3 ).

$\mathrm{M}^{2}$ : De 5 especímenes, uno tiene el protolófulo dirigido hacia adelante y está conectado al anterolófulo (morfotipo 1), en 3 es transverso al protocono (morfotipo 2) y en uno es transverso pero está conectado al entolofo (morfotipo 3). Todos los ejemplares presentan ectolofo: en 2 es corto (morfotipo 2) y en 3 está bien desarrollado (morfotipo 3). En 4 ejemplares el seno es transverso (morfotipo 1) y en uno está inclinado hacia la parte anterior (morfotipo 2). Todos los ejemplares presentan mesolofo: en 4 es medianamente largo alcanzando o sobrepasando (en uno) la mitad del mesoseno (morfotipo 2) y en uno es corto (morfotipo 3). El ectolofo conecta con el mesolofo (morfotipo 1) en 3 ejemplares y en 2 no hay tal conexión (morfotipo 2). El metalófulo es transverso y está conectado al hipocono (morfotipo 1) en 2 ejemplares, es anterior y está conectado al entofolo (morfotipo 2) en 2, y es doble (morfotipo 5) en uno.

Discusión: La morfología de este material es la característica de Megacricetodon collongensis según Daams \& Freudenthal (1988b): en el $\mathrm{M}_{1}$ el anterocónido es simple en casi todos los ejemplares; el desarrollo del mesolófido en los $\mathrm{M}_{1}$ y $\mathrm{M}_{2}$, en los que no hay ejemplares con mesolófido largo y éste es de longitud media, corto o no hay; en el $\mathrm{M}_{2}$ la reducción del anterolófido lingual, que o es corto o no existe; en el $\mathrm{M}^{1}$ : la división del anterocono en todos los ejemplares; sin protuberancia en el anterolófulo; el desarrollo del ectolofo, que en la mayoría de los ejemplares es 
corto o bien desarrollado; el mesolofo, siempre presente aunque generalmente de longitud media o corto; el desarrollo del posterolofo y posteroseno, presente en todos los ejemplares, aunque generalmente reducidos; en el $\mathrm{M}^{2}$ : el desarrollo del protolófulo y ectolofo, siempre presente; la orientación del seno que en general es transverso; la reducción del mesolofo que es generalmente medianamente largo o corto; la existencia o no de conexión entre ectolofo y mesolofo; el desarrollo del metalófulo. La frecuencia del desarrollo de cada uno de estos caracteres en la población de Villaviciosa de Odón es similar al de las poblaciones de Megacricetodon collongensis de Villafeliche 4 A y 4 B de la biozona D y sobre todo a las de Las Planas 4 A, 4B y 4C de la biozona E según Daams \& Freudenthal (1988b). La talla de la población de Villaviciosa de Odón alcanza valores mayores que la de las poblaciones de Villafeliche 4 A y $4 \mathrm{~B}$, y es completamente similar a la de Las Planas 4 A, 4B y $4 \mathrm{C}$, por lo que la mayor similitud la presenta con estas últimas poblaciones de la biozona $\mathrm{E}$. Con respecto a la población de dicha especie del yacimiento madrileño de Somosaguas (Luis \& Hernando, 2000), esta última alcanza valores máximos mayores, mientras que la talla de Megacricetodon collongensis-gersii de Húmera (Cárdaba et al., 2013) es similar. Estos últimos autores (o.c.) consideran que la población del cricétido de Húmera presenta un estado más avanzado que el de Megacricetodon collongensis, en transición con Megacricetodon gersii AGUILAR, 1980, lo que indicaría una edad más reciente dentro de la biozona $\mathrm{E}$. Según Daams et al. (1999b), Megacricetodon gersii, es característica de las biozonas $\mathrm{F} \mathrm{y}_{1}$. Sin embargo, como ya señaló Sesé (2006), esta última especie, que fue definida en el yacimiento del Mioceno Medio de Sansan (Francia) por Aguilar (1980), presenta unas características morfológicas y biométricas que le diferencian de la línea filogenética de talla media a grande del género Megacricetodon en España. Es por ello que en el presente trabajo seguimos el criterio de Daams \& Freudenthal (1988b) que consideran la línea filogenética: Megacricetodon collongensis - Megacricetodon crusafonti - Megacricetodon ibericus, en la que Megacricetodon collongensis está presente en las biozonas D y E, la forma de transición Megacricetodon collongensis-crusafonti en las biozonas $\mathrm{F}$ y $\mathrm{G}_{1}$, Megacricetodon crusafonti en la $\mathrm{G}_{2}$, la forma de transición Megacricetodon crusafonti-ibericus en la $\mathrm{G}_{3}$ y Megacricetodon ibericus en las biozonas $\mathrm{H}$ e I.

\section{Democricetodon sp.}

(Fig. 3: 23 y 24)

Material y Medidas: 1fr. $\mathrm{M}_{1}$ izq. $(\mathrm{L}=--$; $\mathrm{A}=( \pm 1,49) ; 1$ fr. $\mathrm{M}^{1}$ izq. $(\mathrm{L}=--; \mathrm{A}=1,68) ; 1 \mathrm{M}^{2}$ der. $(\mathrm{L}=1,88 ; \mathrm{A}=1,50) ; 1 \mathrm{M}^{3}$ izq. $(\mathrm{L}=1,30 ; \mathrm{A}=1,39)$; $\mathrm{NR}=4 ; \mathrm{NMI}=1$

Descripción y discusión: El material tiene la morfología característica del género Democricetodon. El $\mathrm{M}^{2}$, único molar que está completo, además de un tercer molar superior, es de talla media y entra en la variabilidad de las tallas de Democricetodon larteti (SCHAUB, 1925), Democricetodon jordensi (FREUDENTHAL \& DAAMS, 1988) y Democricetodon lacombai (FREUDENTHAL \& DAAMS, 1988), según los datos de Meulen et al. (2003). Un material tan escaso y fragmentado no es posible asignarlo a ninguna especie con seguridad.

\section{Yacimiento de Leganés}

Orden Lagomorpha BRANDT, 1855

Familia Ochotonidae THOMAS, 1897

Lagopsis verus (HENSEL, 1856)

(Fig. 4: 1 y 2)

Material: 3 D inf.; $6 \mathrm{P}_{3}$ der.; 1 fr. $\mathrm{P}_{3}$ der.; $3 \mathrm{P}_{3}$ izq.; 1 fr. $\mathrm{P}_{3}$ izq.; 2 frs. $\mathrm{P}_{3} ; 37 \mathrm{P} / \mathrm{M}$ inf.; 1 fr. $\mathrm{P} / \mathrm{M}$ inf.; $1 \mathrm{D}$ sup.; 1 fr. maxilar sup. der. con $\mathrm{P}^{2}-\mathrm{P}^{3}-\mathrm{P}^{4}-\mathrm{M}^{1}-\mathrm{M}^{2} ; 1 \mathrm{fr}$. maxilar sup. con $2 \mathrm{P} / \mathrm{M} ; 3 \mathrm{P}^{2}$ der.; 2 frs. $\mathrm{P}^{2}$ der.; $2 \mathrm{P}^{2}$ izq.; $11 \mathrm{P} / \mathrm{M}$ sup.; 5 frs. P/M sup.; 1 fr. $\mathrm{D} ; \mathrm{NR}=86$; $\mathrm{NMI}=7$

Medidas:

\begin{tabular}{|c|c|c|c|c|c|c|c|c|}
\hline \multirow[b]{2}{*}{ DIENTE } & \multirow[b]{2}{*}{$\mathrm{N}$} & \multicolumn{3}{|c|}{ LONGITUD } & \multirow[b]{2}{*}{$\mathrm{N}$} & \multicolumn{3}{|c|}{ ANCHURA } \\
\hline & & MíN. & MED. & MÁX. & & MíN. & MED. & MÁX. \\
\hline $\mathrm{P}_{3}$ & 9 & 1,50 & 1,78 & 1,89 & 9 & 1,65 & 1,77 & 1,98 \\
\hline$P^{2}$ & 1 & - & 1,05 & - & 1 & - & 2,12 & - \\
\hline
\end{tabular}

Descripción y discusión: Adiferencia de Lagopsis peñai, en el $\mathrm{P}_{3}$ el anterocónido y el metacónido no están reducidos. El anterocónido muestra un carácter que claramente le asimila a la especie Lagopsis verus: un anterofléxido labial claro que separa al 

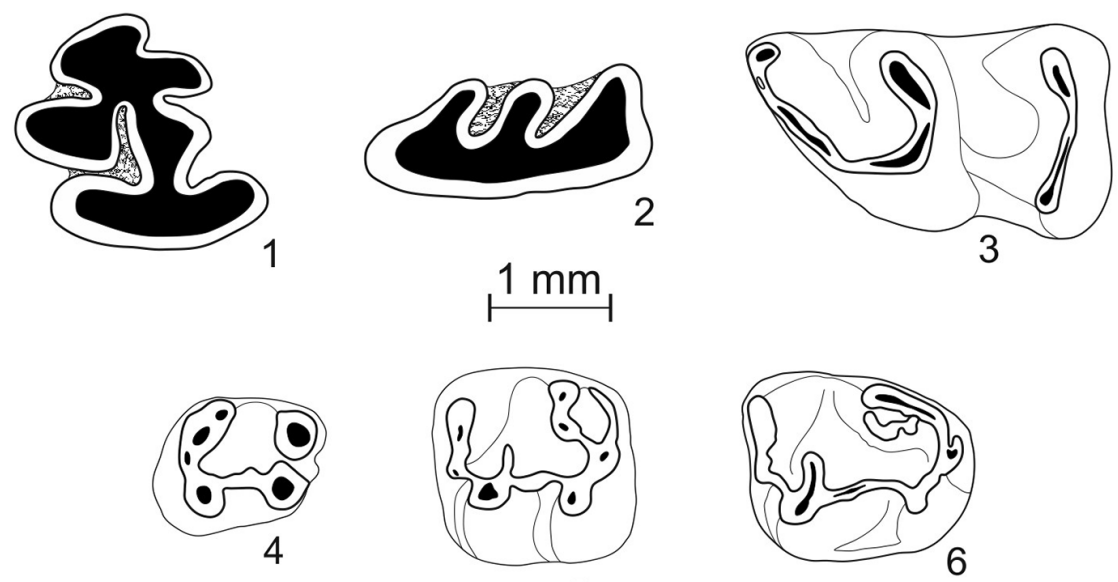

5
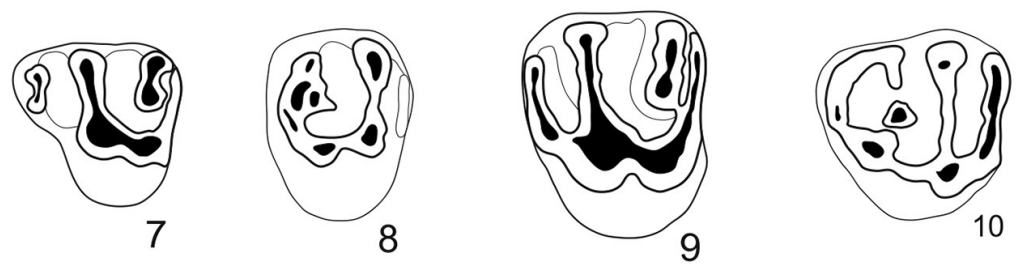

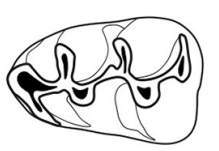

11

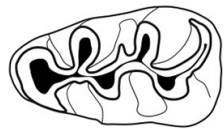

12

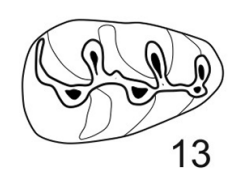

13

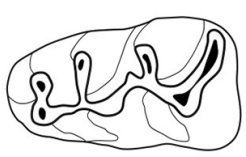

14

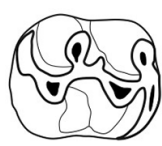

15

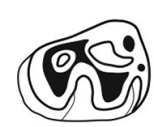

16

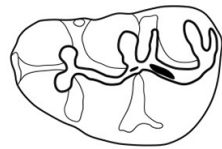

17

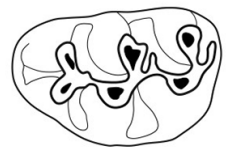

18

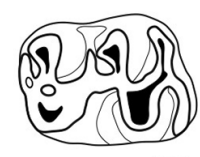

19

Fig. 4.-Dibujos de los dientes de micromamíferos en vista oclusal del yacimiento de Leganés: Lagopsis verus: 1: $P_{3}$ der. (LS-RD 181); 2: $\mathrm{P}^{2}$ der. (LS-RD 9); Galerix cf. exilis: 3: $\mathrm{M}_{1}$ izq. (LS-RD Caja 20); Heteroxerus cf. rubricati: 4: $\mathrm{P}_{4}$ der. (LS-RD 54); 5: $\mathrm{M}_{1-2}$ izq. (LS-RD 57); 6: $\mathrm{M}_{3}$ izq. (LS-RD 94); 7: $\mathrm{D}^{4}$ izq. (LS-RD 125); 8: $\mathrm{P}^{4}$ der. (LS-RD 61); 9: $\mathrm{M}^{1-2}$ izq. (LS-RD 89); 10: $\mathrm{M}^{3}$ der. (LS-RD 58); Megacricetodon collongensis-crusafonti: 11: $M_{1}$ izq. (LS-RD 240); 12: $M_{1}$ izq. (LS-RD 135); 13: $M_{1}$ der. (LS-RD 239); 14: $M_{1}$ izq. (LSRD 142); 15: $M_{2}$ der. (LS-RD 76); 16: $M_{3}$ der. (LS-RD 255); 17: $M^{1}$ izq. (LS-RD 140); 18: $M^{1}$ izq. (LS-RD 137); 19: $M^{2}$ der. (LS-RD 73).

anteroconúlido del anterocónido (López Martínez, 1989). El metacónido es de contorno subcuadrangular con un vértice en su ángulo posterior labial, característico de esta última especie a diferencia de Lagopsis peñai en la que es más pequeño y de contorno redondeado (López Martínez, 1989). La talla entra dentro de la variabilidad de las poblaciones de dicha especie del Aragoniense Superior de la cuenca de Calatayud-Teruel según López Martínez (1989), aunque entre los valores mínimos y medios.
Según López Martínez (1989) Lagopsis peñai da lugar por evolución a Lagopsis verus a través de un aumento de la talla y modificaciones de algunos caracteres como la profundidad del anterofléxido en el $\mathrm{P}_{3} \mathrm{y}$ de los hipoflexos en premolares y molares superiores. Lagopsis peñai se distribuye biostratigráficamente en el Rambliense y Aragoniense Inferior y Medio, y Lagopsis verus en el Aragoniense Superior, edad en cuyo final tiene lugar el último registro conocido hasta ahora 
del género Lagopsis en la Península Ibérica (López Martínez, 1989; Sesé, 2006).

Orden Erinaceomorpha GREGORY, 1910

Familia Erinaceidae FISCHER, 1814

Galerix cf. exilis (BLAINVILLE, 1840)

(Fig. 4.: 3)

Material: 1 fr. $\mathrm{M}_{1}$ der.; $1 \mathrm{M}_{1}$ izq.; 1 fr. $\mathrm{P}^{3}$ izq.; 1 fr. $\mathrm{M}^{1-2}$ izq.; $\mathrm{NR}=4 ; \mathrm{NMI}=1$

Medidas: $1 \mathrm{M}_{1}$ : $\mathrm{L}=3,03 ; \mathrm{A}=1,88$

Descripción y discusión: Comparando este material con el de Galerix exilis de Escobosa de Calatañazor, presenta una morfología similar y la talla entra dentro de la variabilidad de la misma, según los datos de Sesé (1980). Este material es similar al del yacimiento de Villaviciosa de Odón. Ante la escasez y fragmentariedad del material y al faltar piezas dentarias que presenten caracteres con valor diagnóstico de la especie, realizamos su atribución específica con reservas, razón por la que la denominamos Galerix cf. exilis.

Orden Rodentia BOWDICH, 1821

Familia Sciuridae FISCHER DE WALDHEIM, 1817

Heteroxerus cf. rubricati CRUSAFONT, VILLALTA Y TRUYOLS, 1955

(Fig. 4: 7-10)

Material: $4 \mathrm{P}_{4}$ der.; 1 fr. $\mathrm{P}_{4}$ der.; $4 \mathrm{P}_{4}$ izq.; 1 fr. $\mathrm{P}_{4}$ izq.; 1 fr. mandíbula izq. con $\mathrm{M}_{1}-\mathrm{M}_{2} ; 1$ fr. mandíbula der. con $\mathrm{M}_{2}-\mathrm{M}_{3} ; 6 \mathrm{M}_{1-2}$ der.; 4 frs. $\mathrm{M}_{1-2}$ der.; $13 \mathrm{M}_{1-2}$ izq.; 2 frs. $\mathrm{M}_{1-2}$ izq.; $5 \mathrm{M}_{3}$ der.; $6 \mathrm{M}_{3}$ izq.; 1 fr. $\mathrm{M}_{3}$ izq.; 3 frs. $\mathrm{M}$ inf.; $2 \mathrm{D}^{4}$ izq.; 1 fr. $\mathrm{D}^{4}$ izq.; $4 \mathrm{P}^{4}$ der.; $4 \mathrm{P}^{4}$ izq.; 1 fr. $\mathrm{P}^{4}$ izq.; $9 \mathrm{M}^{1-2}$ der.; 3 frs. $\mathrm{M}^{1-2}$ der.; 2 $\mathrm{M}^{1-2}$ izq.; $6 \mathrm{M}^{3}$ der.; 1 fr. $\mathrm{M}^{3}$ der.; $2 \mathrm{M}^{3}$ izq.; 3 frs. $\mathrm{M}$ sup.; 3 frs. $\mathrm{M}$ lado indet.; $\mathrm{NR}=95$; $\mathrm{NMI}=8$

Medidas:

\begin{tabular}{|c|c|c|c|c|c|c|c|c|}
\hline \multirow[b]{2}{*}{ DIENTE } & \multirow[b]{2}{*}{$\mathrm{N}$} & \multicolumn{3}{|c|}{ LONGITUD } & \multirow[b]{2}{*}{$\mathrm{N}$} & \multicolumn{3}{|c|}{ ANCHURA } \\
\hline & & MíN. & MED. & MÁX. & & MíN. & MED. & MÁX. \\
\hline $\mathrm{P}_{4}$ & 7 & 1,13 & 1,28 & 1,38 & 7 & 0,86 & 1,07 & 1,21 \\
\hline$M_{1-2}$ & 18 & 1,41 & 1,54 & 1,70 & 18 & 1,44 & 1,59 & 1,68 \\
\hline$M_{3}$ & 7 & 1,60 & 1,73 & 1,88 & 7 & 1,48 & 1,55 & 1,66 \\
\hline$D^{4}$ & 2 & 1,37 & 1,37 & 1,38 & 2 & 1,27 & 1,30 & 1,34 \\
\hline $\mathrm{P}^{4}$ & 6 & 1,17 & 1,31 & 1,39 & 6 & 1,45 & 1,56 & 1,81 \\
\hline$M^{1-2}$ & 9 & 1,35 & 1,46 & 1,66 & 9 & 1,64 & 1,76 & 1,85 \\
\hline$M^{3}$ & 7 & 1,33 & 1,43 & 1,55 & 7 & 1,42 & 1,51 & 1,57 \\
\hline
\end{tabular}

Descripción y discusión: A pesar de los intentos de diversos autores (Bruijn, 1967; Cuenca Bescós, 1988) por establecer criterios de diferenciación morfológica con caracteres diagnósticos que distingan claramente las especies Heteroxerus rubricati y Heteroxerus grivensis (FORSYTH MAJOR, 1893), la mayor talla de la segunda con respecto a la primera sigue siendo el carácter más importante utilizado generalmente en su determinación taxonómica. Así, en el yacimiento madrileño del Aragoniense Medio madrileño de Húmera, en el que se determinan hasta tres especies: Heteroxerus rubricati, Heteroxerus grivensis y Heteroxerus sp., Cárdaba et al. (2013) señalan que tienen una morfología muy similar y solo se diferencian por la talla. La población de Leganés tiene una talla pequeña que entra dentro de la variabilidad de las poblaciones de Heteroxerus rubricati y es menor que las de Heteroxerus grivensis de Calatayud y Montalbán según los datos de Bruijn (1967) y Cuenca Bescós (1988). Ambas especies son muy comunes en el Mioceno Inferior y Medio de la Península Ibérica, frecuentes asimismo en la mayoría de los yacimientos del Mioceno Medio de Madrid.

\section{Familia Gliridae MUIRHEAD, 1819}

\section{Armantomys sp.}

Material: 1 fr. $\mathrm{M}^{1-2}$ izq.; $\mathrm{NR}=1$; $\mathrm{NMI}=1$.

Descripción y discusión: El único molar disponible presenta la talla y morfología características del género Armantomys: crestas elevadas e inclinadas, ausencia de centrolofos y crestas accesorias, corona dentaria muy hipsodonta. Al estar fragmentado, es insuficiente para determinar la especie.

Familia Cricetidae FISCHER, 1817

Megacricetodon collongensis-crusafonti (MEIN, 1958) - (FREUDENTHAL, 1963) sensu DAAMS \& FREUDENTHAL, 1988b

(Fig. 4: 11-19)

Material: $7 \mathrm{M}_{1}$ der.; 1 fr. $\mathrm{M}_{1}$ der.; $8 \mathrm{M}_{1}$ izq.; 2 frs. $\mathrm{M}_{1}$ izq.; 1 fr. $\mathrm{M}_{1-2}$ izq.; $3 \mathrm{M}_{2}$ der.; 2 frs. $\mathrm{M}_{2}$ der.; $6 \mathrm{M}_{2}$ izq.; 3 frs. $M_{2}$ izq.; $2 M_{3}$ der.; 1 fr. $M_{3}$ der.; $4 M_{3}$ izq.; 1 fr. maxilar sup. con $\mathrm{M}^{1}-\mathrm{M}^{2}$ izq.; $5 \mathrm{M}^{1}$ der.; 2 frs. $\mathrm{M}^{1}$ der.; $6 \mathrm{M}^{1}$ izq.; $3 \mathrm{M}^{2}$ der.; 1 fr. $\mathrm{M}^{2}$ der.; $4 \mathrm{M}^{2}$ izq.; 2 frs. $\mathrm{M}^{2}$ izq.; $1 \mathrm{M}^{3}$ der.; $2 \mathrm{M}^{3}$ izq.; 1 fr. $\mathrm{M}$; $\mathrm{NR}=71 \mathrm{NMI}=10$ 
Medidas:

\begin{tabular}{|c|c|c|c|c|c|c|c|c|}
\hline \multirow[b]{2}{*}{ DIENTE } & \multirow[b]{2}{*}{$\mathrm{N}$} & \multicolumn{3}{|c|}{ LONGITUD } & \multirow[b]{2}{*}{$\mathrm{N}$} & \multicolumn{3}{|c|}{ ANCHURA } \\
\hline & & MíN. & MED. & MÁX. & & MíN. & MED. & MÁX. \\
\hline $\mathrm{M}_{1}$ & 12 & 1,54 & 1,63 & 1,79 & 12 & 0,87 & 0,99 & 1,05 \\
\hline $\mathrm{M}_{2}$ & 8 & 1,18 & 1,22 & 1,32 & 8 & 1,02 & 1,06 & 1,13 \\
\hline $\mathrm{M}_{3}$ & 5 & 0,95 & 1,02 & 1,08 & 5 & 0,75 & 0,81 & 0,85 \\
\hline $\mathrm{M}^{1}$ & 13 & 1,57 & 1,64 & 1,82 & 13 & 1,01 & 1,09 & 1,20 \\
\hline$M^{2}$ & 7 & 1,08 & 1,22 & 1,33 & 7 & 0,97 & 1,01 & 1,08 \\
\hline $\mathrm{M}^{3}$ & 3 & 0,84 & 0,85 & 0,86 & 3 & 0,97 & 0,89 & 0,93 \\
\hline
\end{tabular}

Descripción: El material de Leganés se ha comparado morfológica y biométricamente con el de las diferentes especies del género Megacricetodon de la Cuenca de Calatayud-Teruel descritas por Daams \& Freudenthal (1988b), utilizando los morfotipos que proponen para los diferentes caracteres de los molares:

$\mathrm{M}_{1}$ : De 12 ejemplares que conservan la parte anterior, el anterocónido tiene forma de judía o es ovalado (morfotipo 2) en 6 , y en otros 6 está dividido en forma de 8 (morfotipo 3). De 14 ejemplares, en 7 el mesolófido es corto (morfotipo 3 ) y en otros 7 no existe (morfotipo 4).

$\mathrm{M}_{2}$ : Ningún ejemplar presenta anterolófido lingual (morfotipo 4). De 13 ejemplares, 9 no presentan mesolófido (morfotipo 4) y 4 presentan un corto mesolófido (morfotipo 3).

$\mathrm{M}^{1}$ : El anterocono está perfectamente dividido en todos los ejemplares. En la mayoría de los que no han sufrido desgaste se observa un pequeño cíngulo anterior basal en el borde anterior frente al anterocono (morfotipo 4). Solo un espécimen presenta un anterolófulo labial que es corto (morfotipo 2). De 13 ejemplares: el mesolofo es largo (morfotipo 1) en un ejemplar, mediano alcanzando la mitad del mesoseno en 3 (morfotipo 2), en 7 es corto (morfotipo $3)$ y en 2 no existe (morfotipo 4). En 5 hay ectolofo, que es corto en 4 (morfotipo 2) y largo en uno (morfotipo 3). El metalófulo es posterior en todos los ejemplares, el posteroseno está reducido o ausente y el posterolofo muy corto o ausente (morfotipos 3 y 4 respectivamente).

$\mathrm{M}^{2}$ : De 10 ejemplares, el mesolofo es largo en un ejemplar (morfotipo 1), corto en 5 (morfotipo 3 ) y no existe en 4 (morfotipo 4). En 2 hay un ectolofo largo (morfotipo 3), en 4 es corto (morfotipo 2) y en 4 está ausente (morfotipo 1). El protolófulo es transverso en 4 (morfotipo 2), posterior y transverso en 4 (morfotipo 3) y posterior dirigido hacia atrás en 2 (morfotipo 4). El seno está siempre curvado hacia delante, generalmente ligeramente (morfotipo 2 ) y en 2 ejemplares de forma notable (morfotipo 3 ). En 2 ejemplares el metalófulo es transverso al hipocono (morfotipo 1), en 2 está dirigido hacia adelante y conecta con el entolofo (morfotipo 2), en 3 está dirigido hacia atrás y conectado al posterolofo (morfotipo 4) y en 3 es doble (morfotipo 5).

Discusión: La frecuencia en que se presenta la variabilidad de todos estos caracteres en la población de Leganés: división o no del anterocónido en el $\mathrm{M}_{1}$ (en la mitad de la población respectivamente); reducción de mesolófido en molares inferiores y mesolofo en molares superiores; ausencia de anterolófido lingual en $\mathrm{M}_{2}$; división perfecta del anterocono en $\mathrm{M}^{1}$; desarrollo de protolófulo y metalófulo en $\mathrm{M}^{2} \mathrm{y}$ de este último en $\mathrm{M}^{1}$, es completamente similar a la de las poblaciones de Las Planas 5 B y Valalto 1, denominadas por Daams \& Freudenthal (1988b) Megacricetodon collongensis-crusafonti y situadas en la biozona $G_{1}$ es decir, en la parte basal de la biozona G (Daams \& Freudenthal, 1988a). La talla de la población de Leganés es similar a la de Valalto 1. La población de Las Planas 5 B sin embargo presenta una mayor variabilidad, con valores mínimos claramente inferiores a los de Leganés. Con respecto al escaso material recuperado de este taxón en el cercano yacimiento a Leganés de Ramal Parla (Fernández Marrón et al., 2004), la morfología de este último es similar y la talla entra dentro de la variabilidad de la de Leganés.

La denominación de Megacricetodon collongensis-crusafonti propuesta por Daams \& Freudenthal (1988b), fue sustituida por Daams et al. (1999b) por la de Megacricetodon gersii, presente según dichos autores en el Aragoniense Superior en las biozonas $\mathrm{F}$ y $\mathrm{G}_{1}$. Esta denominación se encuentra frecuentemente en la literatura para este tipo de poblaciones. Sin embargo, como ya hemos dicho anteriormente, en este trabajo seguimos el criterio de Sesé (2006) según el cual Megacricetodon gersii presenta unas características que le distinguen de la línea filogenética de talla media a grande del género Megacricetodon en España, razón por la cual seguimos la denominación de Megacricetodon collongensis-crusafonti. 


\section{cf. Democricetodon sp.}

Material y Medidas: $1 \mathrm{M}_{3}$ izq. ( $\left.\mathrm{L}=1,33 ; \mathrm{A}=1,14\right)$; $\mathrm{NR}=1 ; \mathrm{NMI}=1$

Descripción y discusión: Entre el material de cricétidos, este $\mathrm{M}_{3}$ tiene una talla claramente mayor que Megacricetodon collongensis-crusafonti determinado en este yacimiento y en general mayor que todas las especies del género Megacricetodon. Entra, sin embargo, en la variación de la talla del $\mathrm{M}_{3}$ de las poblaciones de varias especies del género Democricetodon según los datos de Daams \& Freudenthal (1988b) y Meulen et al. (2003), por lo que lo atribuimos con reservas a dicho género ya que es una pieza dentaria que no presenta caracteres diagnóstico relevantes.

\section{Análisis de las asociaciones de micromamíferos: conclusiones}

\section{Bioestratigrafía}

La evolución del género Megacricetodon se ha utilizado para realizar la biostratigrafía del Mioceno en Calatayud-Teruel (Daams \& Freudenthal, 1988 a y b). La especie Megacricetodon collongensis se registra durante el Aragoniense Medio en las biozonas D y E, según Daams \& Freudenthal (1988a), que se correlacionan con la unidad MN 5 (Mein, 1975). La población de Megacricetodon collongensis de Villaviciosa de Odón, de morfología y talla completamente similares a las poblaciones de dicha especie de los yacimientos del área de Calatayud-Teruel de Las Planas 4 A, 4B y 4C que se sitúan en la biozona E (Daams \& Freudenthal, 1988 a y b), permite asignar el yacimiento al final del Aragoniense Medio. Las dataciones magnetostratigráficas realizadas en la cuenca de Calatayud-Teruel proporcionan unas fechas de la biozona E entre 13,80 y 14,06 Ma (Dam et al., 2006).

Megacricetodon collongensis-crusafonti sensu Daams \& Freudenthal (1988 a y b) se registra en el Aragoniense Superior en las biozonas $\mathrm{F}_{\text {y }} \mathrm{G}_{1}$ según dichos autores (o.c.), que se correlacionan, junto con la biozona $\mathrm{G}_{2}$, en la que se registran Megacricetodon crusafonti y Megacricetodon minor, con la unidad MN 6 (Mein, 1975). La presencia de Megacricetodon collongensis-crusafonti en el yacimiento de Leganés, con una talla y una morfología totalmente similares a la población de dicho taxón del yacimiento de Valalto 1 , hace que el yacimiento madrileño se pueda atribuir a la base de la biozona $G$, biozona $\mathrm{G}_{1}$ según Daams \& Freudenthal (1988 a y b). Las dataciones magnetostratigráficas realizadas en la cuenca de Calatayud-Teruel proporcionan unas fechas para la biozona $\mathrm{G}_{1}$ de 13,55 - 13,56 Ma (Dam et al., 2006).

La línea filogenética: Lagopsis peñai - Lagopsis verus también es interesante desde los puntos de vista evolutivo y biostratigráfico. Lagopsis peñai del Rambliense y Aragoniense Inferior y Medio es sustituida por evolución por Lagopsis verus al inicio del Aragoniense Superior (biozona F) y tiene lugar su último registro conocido hasta ahora al final de dicho periodo (biozona $\mathrm{G}_{3}$ ) (López Martínez, 1989; Sesé, 2006). La presencia de Lagopsis peñai en Villaviciosa de Odón corrobora por tanto su edad del Aragoniense Medio y la de Lagopsis verus en Leganés su atribución al Aragoniense Superior.

En el yacimiento de Villaviciosa de Odón se han encontrado también varios dientes atribuibles al artiodáctilo del género Caenotherium. Este taxón tiene también interés biostratigráfico y corrobora la atribución del yacimiento al Aragoniense Medio ya que, según Soria et al. (2000), en las faunas del Mioceno de Madrid, este género, representado por la especie Caenotherium miocaenicum CRUSAFONT, VILLALTA y TRUYOLS, 1955 en el Aragoniense Medio, desaparece al final de dicho periodo y ya no se registra en las de la biozona $\mathrm{F}$ del comienzo del Aragoniense Superior.

En Madrid hay varios yacimientos de la biozona E del Aragoniense Medio: Arroyo del Olivar (Sesé et al., 1985; López Martínez et al., 1987; Soria et al., 2000), Somosaguas (López Martínez et al., 2000 a y b; Luis \& Hernando, 2000; Hernández Fernández et al., 2006), El Cañaveral (Hernández-Ballarín et al., 2010), Casa Montero (López Guerrero et al., 2007) y Húmera (Cárdaba et al., 2013). La mayoría de los taxones que son comunes en estos yacimientos están presentes también en el nuevo yacimiento de Villaviciosa de Odón. La denominación de la población del género Megacricetodon del yacimiento de Húmera (Cárdaba et al., 2013) como Megacricetodon collongensis-gersii, no implica que se trate de un taxón distinto morfológica y biométricamente al de Villaviciosa de Odón, tal como 
se dijo anteriormente, sino de una distinta denominación taxonómica: dichos autores (o.c.) aceptan la línea filogenética Megacricetodon collongensis Megacricetodon gersii propuesta por Daams et al. (1999b) y consideran que la forma de transición Megacricetodon collongensis-gersii del yacimiento de Húmera corresponde a una edad más reciente que la de Megacricetodon collongensis, ambas, sin embargo, en la zona E; mientras que en el presente trabajo, por las razones expuestas anteriormente, aceptamos la línea filogenética Megacricetodon collongensis - Megacricetodon crusafonti propuesta por Daams \& Freudenthal (1988b), según la cual en la zona E sólo estaría presente Megacricetodon collongensis.

Sin embargo, el único yacimiento con micromamíferos del Aragoniense Superior atribuible a la biozona $\mathrm{G}$ es el de Paracuellos 3 (Sesé et al., 1985). Este último yacimiento, caracterizado por la presencia de Megacricetodon crusafonti, está situado por lo tanto biostratigráficamente por encima del nuevo yacimiento de Leganés, muy posiblemente en la biozona $\mathrm{G}_{2}$ en la que es característica dicha especie según Daams et al. (1999b).

\section{Interpretación paleoambiental}

Se han realizado numerosos estudios sobre los roedores del Mioceno, especialmente de las sucesiones faunísticas de la cuenca de Calatayud-Teruel que, además de haber dado a conocer su evolución, lo que ha permitido construir una biozonación muy precisa, también ha dado lugar a un gran conocimiento de las condiciones paleoambientales (temperatura y humedad relativas, y tipo de paisaje) de dicho periodo ya que muchos taxones de roedores son buenos indicadores en tal sentido (Weerd \& Daams, 1978; Meulen \& Bruijn, 1982; Daams \& Meulen, 1984 y 1989; Daams et al., 1988 y 1999b; Meulen \& Daams, 1992). Este tipo de trabajos se han realizado también en la sucesión faunística de micromamíferos del Mioceno Medio de la Cuenca de Madrid (Sesé et al., 1985; López Martínez et al., 1987; López Martínez et al., 2000 a y b; Luis \& Hernando, 2000; Hernández-Ballarín et al., 2011), y utilizando también los macromamíferos (Alberdi et al., 1985; Peláez-Campomanes et al., 2000 y 2003; Soria et al., 2000; Hernández Fernández et al., 2006; Domingo et al., 2008, 2009 y 2012), lo que permite además contrastar los resultados entre ambas cuencas y con otras áreas geográficas más alejadas.

Muchos taxones de roedores miocenos, como han demostrado los mencionados trabajos entre otros muchos, son buenos marcadores paleoambientales atendiendo a varios criterios: las indicaciones en tal sentido de sus representantes actuales, la morfología de su dentición que revela el tipo de alimentación, su asociación con otros taxones indicativos en este sentido, la variación de su frecuencia, aparición o extinción a lo largo del tiempo, etc.

Con respecto a los taxones de micromamíferos que se registran en los yacimientos de Villaviciosa de Odón y Leganés, las indicaciones paleoambientales son las siguientes:

El género Megacricetodon, que es el micromamífero más abundante en ambos yacimientos con un $40 \%$ y $35 \%$ de representación respectivamente (véanse la Tabla 1 y la Figura 5), es cosmopolita según Weerd \& Daams (1978). Aunque posteriormente la línea filogenética de pequeña talla: Megacricetodon minor (LARTET, 1851) - Megacricetodon debruijni FREUDENTHAL, 1968 se interpretó indicadora de clima húmedo, los representantes de la línea de talla media a grande, que es la que se registra en los yacimientos objeto de este trabajo (Megacricetodon collongensis - Megacricetodon crusafonti Megacricetodon ibericus) sigue siendo considerada cosmopolita (Daams et al., 1988; Daams \& Meulen, 1989). El género Megacricetodon es muy abundante en las asociaciones faunísticas del Aragoniense Medio de Madrid, como ya pusieron de manifiesto Sesé et al. (1985) y López Martínez et al. (1987), y más abundante todavía en las del Aragoniense Superior. Esta abundancia ha hecho pensar a dichas autoras (o.c.) en un tipo de vida gregario para este cricétido. En cuanto al otro cricétido determinado en ambos yacimientos, Democricetodon sp. en Villaviciosa de Odón y cf. Democricetodon sp. en Leganés, muy escaso en ambos (5\% y 4\% respectivamente), al no haberse podido precisar la especie no se puede saber sus preferencias paleoambientales.

Entre los roedores, el segundo grupo más abundante en el yacimiento de Leganés son los esciúridos del género Heteroxerus (28\%), mientras que en Villaviciosa de Odón dicho grupo es 
Tabla 1.-Abundancia de los taxones de micromamíferos en los yacimientos de Villaviciosa de Odón y Leganés por Número de Restos (NR), Número Mínimo de Individuos (NMI) y porcentaje del NMI (\%NMI)

\begin{tabular}{lcccccc}
\hline TAXONES / YACIMIENTOS & VILLAVICIOSA DE ODÓN & \multicolumn{3}{c}{ LEGANÉS } \\
\hline EDADES & \multicolumn{2}{c}{ ARAGONIENSE MEDIO } & ARAGONIENSE SUPERIOR \\
\hline ZONAS & \multicolumn{2}{c}{ ZONA E } & \multicolumn{3}{c}{ ZONA G1 } \\
\hline NR / NMI / \%NMI & NR & NMI & \%NMI & NR & NMI & $\%$ NMI \\
\hline Lagopsis peñai & 28 & 5 & 22 & & & \\
Lagopsis verus & & & & 86 & 7 & 25 \\
Galerix cf. exilis & 8 & 2 & 9 & 4 & 1 & 4 \\
Miosorex cf. grivensis & 3 & 1 & 5 & & & \\
Heteroxerus cf. rubricati & & & & 95 & 8 & 28 \\
Atlantoxerus sp. & 11 & 2 & 9 & & & \\
Microdyromys sp. & 4 & 1 & 5 & & & \\
Armantomys triscristatus / Armantomys sp. & 1 & 1 & 5 & 1 & 1 & 4 \\
Megacricetodon collongensis & 43 & 9 & 40 & & & \\
Megacricetodon collongensis-crusafonti & & & & 71 & 10 & 35 \\
Democricetodon sp. / cf. Democricetodon sp. & 4 & 1 & 5 & 1 & 1 & 4 \\
TOTAL & 102 & 22 & 100 & 258 & 28 & 100 \\
\hline
\end{tabular}

poco abundante y está representado por el género Atlantoxerus (9\%). Ambos géneros son dos ardillas de la tribu Xerini, con hábitos terrestres, que indican espacios abiertos y un clima seco (Weerd \& Daams, 1978; Daams et al., 1988; Daams \& Meulen, 1989). Al género Heteroxerus se le atribuye un hábitat estepario; su abundancia en las faunas del Mioceno de Madrid parece indicar un ambiente árido con escasa cobertura vegetal (López Martínez et al., 1987).

Los lagomorfos son muy abundantes en ambos yacimientos y en porcentajes muy similares. El segundo micromamífero en abundancia en el yacimiento de Villaviciosa de Odón es Lagopsis peñai (22\%) y en Leganés Lagopsis verus es el tercero (25\%). El género Lagopsis es un lagomorfo que por su distribución fundamentalmente meridional en Europa durante el Mioceno se considera un taxón termófilo que tiene preferencia por climas relativamente cálidos y los biotopos más húmedos (Mein, 1984). Es un taxón con una dentición hipsodonta especializada en vegetación dura (López Martínez et al., 1987) al que se atribuye un hábitat ripícola o próximo al agua (López Martínez, 1989). Muy abundante en el Rambliense y Aragoniense Inferior y Medio en España, su abundancia disminuye en el
Aragoniense Superior, desapareciendo al final de dicho periodo (Mein, 1984; López Martínez et al., 1987; Sesé, 2006).

Los glíridos son poco abundantes y están representados por Microdyromys sp. (5\%) y Armantomys tricristatus (5\%) en Villaviciosa de Odón y Armantomys sp. (4\%) en Leganés.

El género Microdyromys por su morfología dentaria es omnívoro y, aunque fundamentalmente de hábitat forestal, también se encuentra en espacios abiertos (Meulen \& Bruijn, 1982), por lo que no se le puede adscribir a un tipo de medio concreto, boscoso o abierto. Se considera un taxón cosmopolita y termófilo indicador de altas temperaturas (Daams \& Meulen, 1984 y 1989; López Martínez et al., 1987; Daams et al., 1988). De amplio registro en el Rambliense y Aragoniense desapareció al final del Vallesiense (López Martínez et al., 1987 y 2000a; Sesé, 2006). El género Armantomys tiene una dentición hipsodonta, característica de un mamífero herbívoro muy especializado en vegetación dura de tipo xerofítico, por lo que se le atribuye un hábitat abierto estepario en un clima seco (Weerd \& Daams, 1978; Meulen \& Bruijn, 1982; Daams \& Meulen, 1984 y 1989; Meulen \& Daams, 1992). López Martínez et al. (2000a) le atribuyen un 


\section{VILLAVICIOSA DE ODÓN}
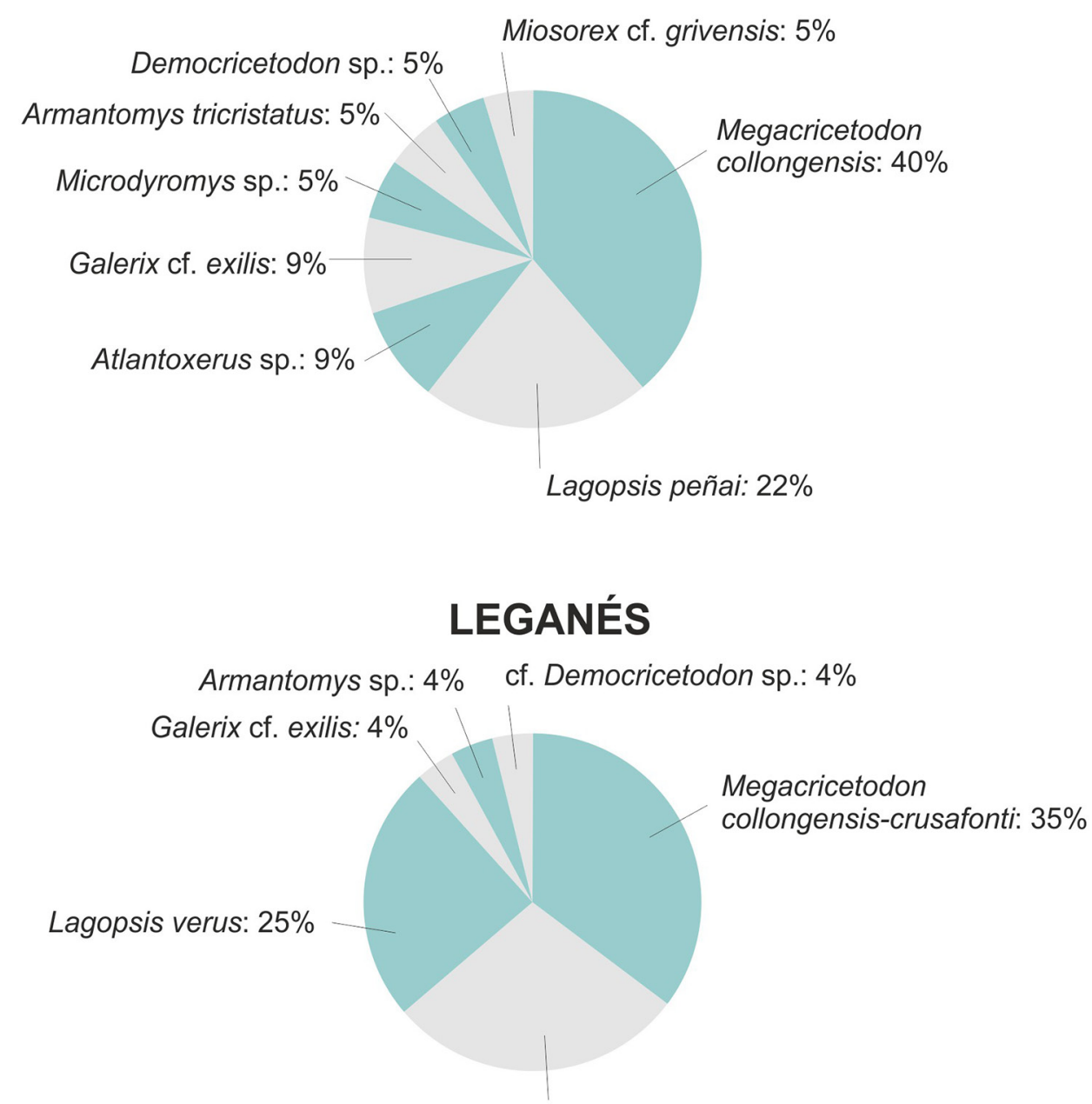

Heteroxerus cf. rubricati: $28 \%$

Fig. 5.-Abundancia porcentual a partir del NMI de los taxones de micromamíferos de los yacimientos de Villaviciosa de Odón y Leganés.

hábitat de estepa cálida por su frecuente asociación con los géneros Lagopsis y Heteroxerus (como es el caso del yacimiento de Leganés). Muy frecuente y generalmente abundante durante el Rambliense y Aragoniense, se extingue el final de dicho periodo (López Martínez et al., 1987; Sesé, 2006).

En cuando a los insectívoros, están escasamente representados en ambos yacimientos: Galerix cf. exilis con un $9 \%$ en Villaviciosa Odón y un $4 \%$ en Leganés, y Miosorex cf. grivensis en el primer yacimiento con un 5\%. Según Hernández Fernández et al. (2006), la primera especie se considera que está relacionada con la actual rata lunar o gimnuro
(Echinosorex gymnura) que habita en climas tropicales en el sudeste asiático.

Teniendo en cuentas todas estas indicaciones, en el yacimiento Villaviciosa de Odón, la presencia de Atlantoxerus sp. (9\%) y Armantomys tricristatus (5\%), aunque ninguno de ellos es abundante, indica la existencia de un medio abierto estepario y un clima seco árido. En todo caso, no se registran taxones que indiquen zonas boscosas y condiciones de cierta humedad relativa. Lagopsis peñai, muy abundante $(22 \%)$ posiblemente ocuparía las zonas próximas al agua del entorno. Tanto este lagomorfo como Microdyromys sp. (5\%) son indicadores termófilos. 
En resumen, la asociación de micromamíferos de Villaviciosa de Odón sugiere un medio fundamentalmente abierto con predominio de la vegetación dura de tipo estepario, en el que algunos taxones como Lagopsis peñai ocuparían los biotopos más próximos al agua o más húmedos, y un clima seco y relativamente cálido.

El yacimiento de Somosaguas Sur, de edad similar a la de Villaviciosa de Odón, con una composición faunística casi idéntica, excepto por el registro en el primer yacimiento de Heteroxerus grivensis y una nueva especie de cricétido: Cricetodon soriae LÓPEZ-MARTÍNEZ, CÁRDABA, SALESA, HERNÁNDEZ FERNÁNDEZ, CUEVAS-GONZÁLEZ \& FESHARAKI (Hernández Fernández et al., 2006), es muy diferente en cuanto a la abundancia de los taxones, según los datos de Luis \& Hernando (2000). Mientras en Villaviciosa de Odón los lagomorfos son el segundo taxón más abundante $(22 \%)$, en Somosaguas solo representan el 2\%. Además, mientras en Somosaguas el género Fahlbuschia (que Meulen et al., 2003 consideran sinonimia del género Democricetodon), es el segundo taxón en abundancia, en Villaviciosa de Odón está escasamente representado (5\%). Sin embargo, la abundancia relativa de los taxones de Villaviciosa de Odón es parecida a la de Arroyo del Olivar, según datos de Luis \& Hernando (2000), yacimiento de edad similar en el que los lagomorfos son también muy abundantes con el mismo porcentaje (22\%), aunque los géneros Fahlbuschia (=Democricetodon) y Armantomys tienen también mayor representación en éste. Las diferencias de abundancia de los distintos taxones de micromamíferos entre unos y otros yacimientos, cuya composición taxonómica y edades son similares, pueden ser debidas, tal como señalan Luis \& Hernando (2000), a los distintos condicionamientos que suponen las propias diferencias paleofisiográficas de su distinta ubicación, la proximidad de los taxones o no a su biotopo preferencial, origen de la acumulación de los restos, etc. En el yacimiento de Somosaguas Sur, Luis \& Hernando (2000), del estudio de los microvertebrados concluyen que se infiere la existencia de un clima cálido y árido, y un medio predominantemente abierto en el que hay un predominio de vegetación dura, aunque con un desarrollo vegetal que permitiría el mantenimiento de dicha fauna, y comparan el biotopo con las sabanas tropicales o subtropicales actuales. Asimismo el análisis de la estructura de los tamaños corporales de la comunidad de mamíferos sugiere un medio de tipo sabana (Perales et al., 2009) $y$, por otra parte, el estudio de los patrones de edad de muerte de Democricetodon larteti, que indican una gran mortandad de individuos juveniles, se asocia a eventos de gran aridez (Torroba et al., 2010).

En el yacimiento de Leganés, el género Megacricetodon, representado por Megacricetodon collongensis-crusafonti, una forma más evolucionada que Megacricetodon collongensis de Villaviciosa de Odón, sigue siendo el taxón más abundante y con un porcentaje de representación (35\%) bastante similar; lo mismo sucede con Lagopsis verus (que sustituye por evolución a Lagopsis peñai de este último yacimiento) que, aunque es el tercer taxón en abundancia, está representado en un porcentaje similar (25\%). La mayor diferencia entre uno y otro yacimiento radica en el registro en Leganés de Heteroxerus cf. rubricati, segundo taxón en abundancia (28\%), que indica un hábitat estepario y clima seco. Armantomys sp., aunque escasamente representado (4\%), tiene el mismo tipo de preferencias paleoambientales. En Leganés también destaca la abundancia de Lagopsis verus como indicador termófilo. Por todo ello, en el yacimiento de Leganés la asociación faunística es más claramente indicativa de un medio predominantemente abierto estepario y también de la aridez del clima.

Con estos datos, sin embargo, no se puede hablar en rigor de una mayor aridez del clima en Madrid durante la biozona $\mathrm{G}_{1}$, a la que corresponde el yacimiento de Leganés, con respecto a la biozona $\mathrm{E}$, a la que corresponde Villaviciosa de Odón, ya que hay que tener en cuenta los datos disponibles de otros yacimientos de esta última biozona: Somosaguas y Arroyo del Olivar. Así, sumando los porcentajes de representación de los géneros Atlantoxerus, Heteroxerus y Armantomys, que son los mayores indicadores de este tipo de condiciones ambientales, en Leganés supone un 32\% en total; este porcentaje, aunque muy superior a su representación en el yacimiento de Villaviciosa de Odón (14\%) y el de Somosaguas (14\%), es sin embargo muy similar al del yacimiento de Arroyo del Olivar (34\%). Las diferencias pueden ser debidas, por tanto, no solo a su distinta edad, sino a su distinta ubicación geográfica, procesos tafonómicos y de otro tipo, tal como 
se dijo anteriormente. No obstante, sí se puede afirmar que ambas asociaciones, la de Villaviciosa de Odón del Aragoniense Medio y la de Leganés del Aragoniense Superior, corresponden a un tipo de medio y clima parecido puesto que la similitud en su composición taxonómica, y en parte también cuantitativa de algunos de los taxones más abundantes, así parecen indicarlo.

Sobre la base de las asociaciones de micromamíferos del Mioceno Medio de Madrid, Sesé et al. (1985) y López Martínez et al. (1987) ya señalaron la existencia de un medio abierto y árido dominado por vegetación dura, y unas condiciones de clima relativamente cálido y seco durante el Aragoniense Medio y Aragoniense Superior. Alberdi et al. (1985) también constataron la similitud entre las asociaciones del Aragoniense Medio y las del Aragoniense Superior: "Los micromamíferos cambian poco en el tiempo y el espacio: especies equivalentes se sustituyen en el tiempo, mientras que la composición cuantitativa, aunque variable entre los diferentes yacimientos, mantiene en general una gran constancia" (o.c.: p. 101).

Los yacimientos del Aragoniense de Madrid comparables en edad al de Villaviciosa de Odón, y que han sido objeto de numerosos estudios, son los de Somosaguas. La gran riqueza de estos yacimientos (Somosaguas Norte con restos fundamentalmente de macromamíferos y Somosaguas Sur con micromamíferos) de la biozona $\mathrm{E}$, con hasta 23 especies de mamíferos, además de diversas especies de otros grupos de vertebrados terrestres, ha permitido realizar estudios sobre dichos grupos de los que se han obtenido inferencias paleoambientales y climáticas de gran importancia para este periodo: La fauna de macromamíferos presenta la estructura de una comunidad propia de sabana, en la que se combinan espacios abiertos y zonas boscosas asociadas al medio lacustre, en un clima tropical semiárido con estacionalidad hídrica muy marcada (Hernández Fernández et al., 2003 y 2006). Esta variedad de medios abiertos y boscosos que encuentran estos autores (o.c.) a partir del estudio de los macromamíferos, contrasta con los resultados obtenidos a partir de los taxones de micromamíferos indicados anteriormente por Luis \& Hernando (2000) en Somosaguas Sur, según los cuales sería un tipo de medio predominantemente abierto. Alberdi et al. (1985) ya señalaban las aparentes discrepancias entre las indicaciones paleoambientales inferidas a partir de las asociaciones de micromamíferos y macromamíferos del Aragoniense Medio y Superior en Madrid ya que, así como en las de micromamíferos hay una cierta continuidad en su composición a lo largo del tiempo, que indica asimismo una continuidad de las condiciones paleoambientales, tal como se ha dicho más arriba, las inferidas a partir de los macromamíferos cambian radicalmente entre los yacimientos, tanto en el tiempo como en el espacio, e indican cambios en la humedad y en la vegetación. Por otra parte, Hernández Fernández et al. (2003 y 2006) señalan que, aunque los mamíferos indican la existencia de condiciones tropicales, a partir del análisis realizado en la sucesión de yacimientos de macromamíferos del Aragoniense de España, se detecta que la secuencia de Somosaguas, de una edad estimada de unos $14 \mathrm{Ma}$, se sitúa en un momento de brusco enfriamiento y de aridez progresiva (aridez que también se muestra en los micromamíferos según Luis \& Hernando, 2000), que se correlaciona con el cambio hacia el enfriamiento del clima a escala global, inferido a partir de estudios isotópicos realizados en faunas marinas y en menor medida de registros en medios continentales, que acaeció en el Mioceno Medio hace entre 15 y 13 Ma, lo que también señalaron López Martínez et al. (2000b) sobre dicho yacimiento. Estudios geoquímicos sobre isótopos estables de oxígeno y carbono del esmalte dental de grandes herbívoros de Somosaguas (Hernández Fernández et al., 2006; Domingo et al., 2008, 2009), y en general de grandes herbívoros de los yacimientos del Aragoniense Medio y Superior de Madrid (Domingo et al., 2012), corroboran, en efecto, que se observa esta crisis paleoclimática con la caída de la temperatura (que incluso llegan a cuantificar en unos $6^{\circ}$ ), que tuvo lugar a escala global durante la biozona E del Aragoniense Medio, así como una caída de la humedad con un cambio hacia condiciones más áridas. Cuevas González (2005) a partir de la paleontología, estratigrafía y sedimentología de Somosaguas dedujo que en la Cuenca de Madrid el máximo de aridez durante el Aragoniense tuvo lugar más probablemente en la biozona $\mathrm{E}$, tal y como Meulen \& Daams (1992) habían inferido 
anteriormente en la cuenca de Calatayud-Teruel a partir del estudio de los micromamíferos. Aunque Hernández Fernández et al. (2006) se muestran en principio de acuerdo con dicha hipótesis, basándose en la riqueza de especies de roedores propias de ambiente árido en dicha zona, estudios más generalistas teniendo en cuenta también los macromamíferos indican las condiciones generales de clima árido, con diversas fluctuaciones y momentos de máxima aridez en las últimas biozonas del Aragoniense Medio y primeras del Aragoniense Superior.

Con respecto a las condiciones paleoambientales de la biozona $G_{1}$ en la que se sitúa el yacimiento de Leganés, no hay datos comparables de yacimientos con micromamíferos de la misma edad en la Cuenca de Madrid, pero sí hay datos en la de Calatayud-Teruel. Uno de los estudios realizados con roedores en esta cuenca es el de Meulen \& Daams (1992) que consideran que, en la biozona $\mathrm{G}_{1}$, con respecto a biozonas anteriores, los glíridos, entre los cuales hay taxones propios de bosque (géneros Myoglis, Muscardinus), están más diversificados y que la especie de la línea de pequeña talla Megacricetodon minor es, asimismo, habitante de bosque, por lo que concluyen que hay un aumento de la humedad en este periodo. En cuanto a la curva de temperatura, según dichos autores (o.c.) parece que no hay cambios apreciables a partir del enfriamiento que se registra en la zona $\mathrm{E}$. En el yacimiento de Leganés, sin embargo, no se registra ninguna de los mencionados taxones y la asociación es bastante similar a la del yacimiento de Villaviciosa de Odón, lo que sugiere un medio que seguiría siendo fundamentalmente abierto y el clima árido como en la biozona $\mathrm{E}$, de forma similar a lo que ya señalaron Sesé et al. (1985), Alberdi et al. (1985) y López Martínez et al. (1987) para las asociaciones del Aragoniense Medio y Superior de Madrid. Lo mismo puede decirse de Paracuellos 3 , yacimiento en el que tampoco se registra ninguno de dichos taxones de las asociaciones de Calatayud-Teruel, y que no presenta nuevos taxones diferentes con respecto a biozonas anteriores, excepto el ya indicado, Megacricetodon crusafonti, que no es un nuevo taxón sino que corresponde a un estadio más evolucionado dentro de la línea de Megacricetodon collongensis - Megacricetodon crusafonti. No obstante, Alberdi et al. (1985) indican que la asociación de macromamíferos de Paracuellos 3 también corresponde a un paisaje abierto con cierta aridez en un clima cálido, aunque relativamente más húmedo que en el Aragoniense Medio. Por otra parte, los resultados del estudio realizado por Hernández Fernández et al. (2006), mencionado anteriormente, corroboran la hipótesis de la continuidad de las condiciones ambientales de aridez basadas en mamíferos de Madrid entre el final del Aragoniense Medio y comienzos del Aragoniense Superior.

Por último, y abundando en la tendencia aquí señalada hacia la aridez climática a partir del Aragoniense Medio, hay que señalar que en los cercanos yacimientos a Leganés de Ramal Parla, Ramal Fuenlabrada y EDAR Culebro mencionados anteriormente, en los que se registra flora, y, coronando la secuencia fosilífera, una fauna de micromamíferos que la supradata en el Aragoniense Superior (zonas $\mathrm{F}_{\text {o }} \mathrm{G}_{1}$ ) (Fernández Marrón et al., 2004), se concluye que a partir de las plantas fósiles se observa una evolución paleoambiental en dicha sucesión aragoniense con una tendencia al desarrollo de zonas esteparias en el tramo superior con respecto a las áreas palustres-lacustres del inferior.

\section{AGRADECIMIENTOS}

Las intervenciones paleontológicas se realizaron en cumplimiento de la Ley 18/1998 de 9 de Julio de Patrimonio Histórico de la Comunidad de Madrid (que actualmente ha pasado a ser la Ley 3/2013 de 18 de Junio) y fueron autorizadas, gestionadas y supervisadas por la Dirección General de Patrimonio Histórico Artístico de la Consejería de Educación. Según el artículo 30 de esta última ley, los permisos son: 1) Área de Planeamiento Integrado "Sector I". La Tenería. Villaviciosa de Odón". Expediente: 1033/00. Permiso expedido con fecha 12 de enero de 2001. Referencia: 09/008866.0/01; 2) Emisario Variante de Parla hasta nueva EDAR Fuenlabrada-Culebro. REF 328.00. Permiso expedido con fecha 14 de julio de 2000. Referencia: 09/249000.5/00.

Los directores de la prospección fueron el arqueólogo Carlos Barrio Aldea, a quien agradecemos su colaboración, y uno de los firmantes de este trabajo (JC JR). Nuestro agradecimiento también al arqueólogo Raúl Arribas Domínguez por la colaboración prestada, a Enrique Soto por su valiosa ayuda en la puesta a punto de las figuras, y a Javier Murelaga y Francisco Javier Ruiz-Sánchez, los revisores del manuscrito, que con sus sugerencias han contribuido a su mejora. 


\section{Referencias}

Aguilar, J.P. (1980). Nouvelle interpretation de 1'Evolution du genre Megacricetodon au cours du Miocène. Palaeovertebrata, Mémoire Jubilaire en hommage a R. Lavocat: 355-364.

Alberdi, M.T. (ed.) (1985). Geología y paleontología del Terciario continental de la provincia de Madrid. CSICMNCN, Madrid, 105 pp.

Alberdi, M.T.; Morales, J. \& Sesé, C. (1980). Un nuevo yacimiento en el Mioceno de Madrid. II Jornadas de estudios sobre la provincia de Madrid, Diputación Provincial de Madrid, Madrid, 26-31.

Alberdi, M.T.; Jiménez, E.; Morales, J. \& Sesé, C. (1981). Moratines: Primeros Micromamíferos en el Mioceno medio del área de Madrid. Estudios Geológicos, 37: 291-306.

Alberdi, M.T.; Hoyos, M.; Mazo,A.V.; Morales, J.; Sesé, C. \& Soria, D. (1985). Biostratigrafía, paleoecología y biogeografía del Terciario de la provincia de Madrid. In: Geología y paleontología del Terciario continental de la provincia de Madrid. (Alberdi, M.T., ed.), CSIC-MNCN, Madrid, 99-105.

Bruijn, H. de (1967). Gliridae, Sciuridae y Eomyidae (Rodentia, Mammalia) Miocenos de Calatayud (Provincia de Zaragoza, España) y su relación con la bioestratigrafía del área. Boletín del IGME, 78: 187-373.

Calvo, J.M.; Ordóñez, S.; Hoyos, M. \& García del Cura, M.A. (1984). Caracterización sedimentológica de la unidad intermedia del Mioceno de la zona sur de Madrid. Revista de materiales y procesos geológicos, 2: $145-176$.

Calvo, J.P.; Ordóñez, S.; García del Cura, M.A.; Hoyos, M.; Alonso-Zarza, A.M.; Sanz, E. \& Rodríguez Aranda, J.P. (1989). Sedimentología de los complejos lacustres miocenos de la Cuenca de Madrid. Acta Geológica Hispánica, 24 (3-4): 281-298.

Cárdaba, J.A.; Presumido, M.; Fesharaki, O.; Casado, A.I.; Perales, R. \& Muñiz Pérez, M. (2013). New data on the mammalian systematics and biostratigraphy of the middle Miocene from Húmera (Pozuelo de Alarcón, Madrid). Spanish Journal of Palaeontology, 28 (1): 29-42.

Cuenca Bescós, G. (1988). Revisión de los Sciuridae del Aragoniense y del Rambliense en la fosa de Calatayud-Montalbán. Scripta Geologica, 87: $1-116$.

Cuevas-González, J. (2005). Estado actual de los conocimientos paleontológicos y estratigráficos de los yacimientos aragonienses de Somosaguas (Pozuelo de Alarcón, Madrid). Coloquios de Paleontología, 55: 103-123.

Daams, R. (1981). The dental pattern of the dormice Dryomys, Myomimus, Microdyromys and Peridyromys. Utrecht Micropaleontological Bulletins, Special Publication, 3: 1-115.
Daams, R. (1990). Hypsodont Myomiminae (Gliridae, Rodentia) from the Miocene and the OligoceneMiocene boundary interval of Spain. Scripta Geologica, 95: 1-62.

Daams, R. \& Meulen, A.J. van der (1984). Paleoenvironmental and paleoclimatic interpretation of Micromammal faunal successions in the Upper Oligocene and Miocene of North CentralSpain.Paléobiologie continentale, 14 (2): 241-257.

Daams, R.\&Freudenthal,M.(1988a). Synopsis oftheDutchSpanish collaboration program in the Aragonian type area, 1975-1986. In: Biostratigraphy and paleoecology of the Neogene micromammalian faunas from the Calatayud-Teruel Basin (Spain). (Freudenthal, M., ed.). Scripta Geologica, Special Issue: 3-18.

Daams, R. \& Freudenthal, M (1988b). Cricetidae (Rodentia) from the type-Aragonian: the genus Megacricetodon. In: Biostratigraphy and paleoecology of the Neogene micromammalian faunas from the Calatayud-Teruel Basin (Spain). (Freudenthal, M., ed.). Scripta Geologica, Special Issue: 39-132.

Daams, R.; Freudenthal, M. \& Meulen, A.J. van der (1988). Ecostratigraphy of micromammal faunas from the Neogene of Spain. In: Biostratigraphy and paleoecology of the Neogene micromammalian faunas from the Calatayud-Teruel Basin (Spain). (Freudenthal, M., ed.). Scripta Geologica, Special Issue: 287-302.

Daams, R. \& Meulen, A.J. van der (1989). Implicaciones paleoclimáticas y paleoecológicas de las sucesiones de micromamíferos en el Neógeno de la cuenca de Calatayud-Teruel. In: Nuevas Tendencias, Paleontología (Aguirre, E., ed.), CSIC, Madrid, 14 (2): 223-235.

Daams, R.; Meulen, A.J. van der; Álvarez Sierra, M.A.; Peláez-Campomanes, P. \& Krijgsman, W. (1999a). Aragonian stratigraphy reconsidered, and a re-evaluation of the middle Miocene mammal biochronology in Europe. Earth and Planetary Science Letters, 165: 287-294. http://dx.doi.org/10.1016/ S0012-821X(98)00273-8.

Daams, R.; Meulen, A.J. van der; Álvarez Sierra, M.A.; Peláez-Campomanes, P.; Calvo, J.P.; Alonso Zarza, M.A. \& Krijgsman, W. (1999b). Stratigraphy and sedimentology of the Aragonian (Early to Middle Miocene) in its type area (North-Central Spain). Newsletters on Stratigraphy, 37 (3): 103-139.

Dam, J. van; Aziz, H.A.; Álvarez Sierra, M.A.; Hilgen, F.J.; Hoek Ostende, L.W. van den; Lourens, L.J.; Mein, P.; van der Meulen, A.J. \& Peláez-Campomanes, P. (2006). Long-period astronomical forcing of mammal turnover. Nature, 443: 687-691. http://dx. doi.org/10.1038/nature05163.

Domingo, L.; Cuevas-González, J.; Grimes, S.T. \& López Martínez, N. (2008). Reconstrucción paleoclimática y paleoecológica del yacimiento de Somosaguas (Mioceno medio, Cuenca de Madrid) mediante el análisis geoquímico del esmalte dental de herbívoros. Palaeontologica Nova, SEPAZ, 8: 187-200. 
Domingo, L.; Cuevas-González, J.; Grimes, S.T.; Hernández Fernández, M. \& López Martínez, N. (2009). Multiproxy reconstruction of the palaeoclimate and palaeoenvironment of the Middle Miocene Somosaguas site (Madrid, Spain) using herbivore dental enamel. Palaeogeography, Palaeoclimatology, Palaeoecology, 272: 53-68. http://dx.doi.org/10.1016/j.palaeo.2008.11.006.

Domingo, L.; Koch, P.L.; Grimes, S.T.; Morales, J. \& López Martínez, N. (2012). Isotopic paleoecology of mammals and the Middle Miocene Cooling event in the Madrid Basin (Spain). Palaeogeography, Palaeoclimatology, Palaeoecology, 338-341: 98-113. http://dx.doi.org/10.1016/j.palaeo.2012.04.026.

Fernández Marrón. M.T.; Fonollá Ocete, J.F. \& Jiménez Rodrigo, J.C. (2002). Primeros datos Paleobotánicos y Palinológicos de una flora miocénica procedente de la unidad intermedia en el sector central de la Cuenca de Madrid. Geogaceta, 31: 177-180.

Fernández Marrón. M.T.; Fonollá Ocete, J.F.; Sesé Benito, C. \& Jiménez Rodrigo, J.C. (2004). Estudio paleoambiental de nuevos yacimientos de plantas y vertebrados de la «Unidad Intermedia» del Mioceno Medio de la Cuenca de Madrid. Revista Española de Paleontología, 19 (2): 199-213.

García Paredes, I. (2006). Patrones evolutivos de los Gliridae (Rodentia, Mammalia) del Mioceno Inferior y Medio del área tipo del Aragoniense (Cuenca de Calatayud-Montalbán). Tesis Doctoral, Universidad Complutense de Madrid, 676 pp.

Gibert, J. (1975). Distribución bioestratigráfica de los Insectívoros del Mioceno en el NE de España. Biotopos, comparación de cuencas y localidades. Relaciones faunísticas con América del Norte. Acta Geológica Hispánica, 10 (5): 167-169.

Hernández-Ballarín, V.; Oliver, A.; García-Paredes, I. \& Peláez-Campomanes, P. (2010). Estudio preliminar de la fauna de roedores del yacimiento mioceno de El Cañaveral (Madrid, España). In: VIII Encuentro de Jóvenes Investigadores en Paleontología (Moreno-Azanza, M.; Díaz-Martínez, I.; Gasca, J.M.; Melero-Rubio, M.; Rabal-Garcés, R. \& Sauqué, V., eds.). Cidaris, 30: 151-156.

Hernández-Ballarín, V.; Oliver, A. \& Peláez-Campomanes, P. (2011). Revisión de las asociaciones de mamíferos del tránsito Aragoniense medio y superior de la Cuenca de Madrid. In: Viajando a Mundos Pretéritos (PérezGarcía, A.; Gascó, F.; Gasulla, J.M. \& Escaso, F., eds.). Ayuntamiento de Morella, Morella, Castellón, 173-182.

Hernández Fernández, M.; Salesa, M.J.; Sánchez, I.M. \& Morales, J. (2003). Paleoecología el género Anchitherium von Meyer, 1834 (Equidae, Perissodactyla, Mammalia) en España: evidencias a partir de las faunas de macromamíferos. Coloquios de Paleontología, Vol. Ext. 1: 253-280.

Hernández Fernández, M.; Cardaba, J.A.; CuevasGonzález, J.; Fesharaki, O.; Salesa, M.J.; Corrales, B.; Domingo, L.; Elez, J.; López Guerrero, P.;
Sala-Burgos, N.; Morales, J.; López Martínez, N. (2006). Los yacimientos de vertebrados del Mioceno medio de Somosaguas (Pozuelo de Alarcón, Madrid): implicaciones paleoambientales y paleoclimáticas. Estudios Geológicos, 62 (1): 263-294. http://dx.doi. org/10.3989/egeol.0662126.

Herráez, E; García Paredes, I.; Peláez-Campomanes, P. \& Morales, J. (2006). Los Nogales, nueva fauna de vertebrados del Mioceno medio de Madrid. Estudios Geológicos, 62 (1): 257-262. http://dx.doi. org/10.3989/egeol.0662125.

Hoek Ostende, L.W. van den (2001). A revised generic classification of the Galericini (Insectivora, Mammalia) with some remarks on their Palaeobiogeography and phylogeny. Geobios, 34 (6): 681-695. http://dx. doi.org/10.1016/S0016-6995(01)80029-2.

Hoek Ostende, L.W. van den (2003). Insectivores (Erinaceomorpha, Soricomorpha, Mammalia) from the Ramblian of the Daroca-Calamocha area. Coloquios de Paleontología, Vol. Extr.: 281-310.

Hoek Ostende, L.W. van den \& Furió, M. (2005). Spain. In: The Fossil Record of the Eurassian Neogene insectivores (Erinaceomorpha, Soricomorpha, Mammalia). Part I. (Hoek Ostende, L.W. van den; Doukas, C.S. \& Reumer, J.W.F., eds.). Scripta Geologica, Special issue, 5: 149-284.

Hoyos, M.; Junco, F.; Plaza, J.M.; Ramírez, A.\& Ruiz, J. (1985). El Mioceno de Madrid. In: Geología y Paleontología del Terciario Continental de la Provincia de Madrid (Alberdi, M.T., ed.). CSIC-MNCN, Madrid, 9-16.

Jong, F. de (1988). Insectivora from the Upper Aragonian and the Lower Vallesian of the Daroca-Villafeliche area in the Calatayud-Teruel Basin (Spain). In: Biostratigraphy and paleoecology of the Neogene micromammalian faunas from the Calatayud-Teruel Basin (Spain). (Freudenthal, M., ed.). Scripta Geologica, Special Issue: 253-258.

Junco, F. \& Calvo, J.P. (1983). La Cuenca de Madrid In: Libro Jubilar J.M. Ríos. Geología de España. Tomo I, ITGE, 534-542.

López-Guerrero, P.; Oliver Pérez, A.; Álvarez Sierra, M.A.; García Paredes, I.; van den Hoek Ostende, L.W. \& Peláez-Campomanes de Labra, P. (2007). Paleontología del yacimiento de vertebrados Aragoniense de Casa Montero (Madrid). Cantera Paleontológica, 53: 247-254.

López Martínez, N. (1989). Revisión sistemática y bioestratigráfica de los Lagomorpha (Mammalia) del Terciario y Cuaternario de España. Memorias del Museo Paleontológico de la Universidad de Zaragoza, 3 (3): $343 \mathrm{pp}$.

López Martínez, N.; Sesé, C. \& Sanz, J.L. (1977). La Microfauna (Rodentia, Insectivora, Lagomorpha y Reptilia) de las fisuras del Mioceno medio de Escobosa de Calatañazor (Soria, España). Acta Geológica Hispánica, 12 (1/3): 60-68. 
López Martínez, N.; Sesé, C. \& Herráez, E. (1987). Los yacimientos de micromamíferos del área de Madrid. Boletín Geológico y Minero, 97 (3): 159-176.

López Martínez, N.; Elez Villar, J.; Hernando Hernando, M.; Luís Cavia, A.; Minués Gandú, D.; Polonio Martín, I.; Salesa, M.; Mazo, A. \& Sánchez, I. (2000a). Los vertebrados fósiles de Somosaguas (Pozuelo de Alarcón, Madrid). In: Patrimonio Paleontológico de la Comunidad de Madrid (Morales, J.; Nieto, M.;Amezua, L.; Fraile, S.; Herráez, E.; Peláez-Campomanes, P.; Salesa, M.J.; Sánchez, I.M. \& Soria, D., eds.). Arqueología, Paleontología y Etnografía, 6: 130-140.

López Martínez, N.; Elez, J.; Hernando, J.M.; Luis, A.; Mazo, A., Mínguez Gandu, D.; Morales, J.; Polonio, I.; Salesa, J.M. \& Sánchez, I. (2000b). Los vertebrados fósiles de Somosaguas (Pozuelo, Madrid). Coloquios de Paleontología, 51: 69-86.

López Olmedo, F.; Díaz de Neira, A.; Martín Serrano, A.; Calvo, J.P.; Morales, J. \& Peláez-Campomanes, P. (2003). Estratigrafía de los depósitos arcósicos en el sector occidental de la Cuenca de Madrid. Geotemas, 5: 141-146.

López Olmedo, F.; Díaz de Neira, A.; Martín Serrano, A.; Calvo, J.P.; Morales, J. \& Peláez-Campomanes, P. (2004). Unidades estratigráficas en el registro sedimentario neógeno del sector occidental de la Cuenca de Madrid. Revista de la Sociedad Geológica de España, 17: 87-101.

Luis, A. \& Hernando, J.M. (2000). Los microvertebrados del Mioceno medio de Somosaguas Sur (Pozuelo de Alarcón, Madrid, España). Coloquios de Paleontología, 51: 87-136.

Mein, P. (1975). Résultats du groupe de travail des vertébrés. In: Report on Activity of the RCMNS Working Groups (1971-1975) (Senes, J., ed.), Bratislava, 78-81.

Mein, P. (1984). Composition quantitative des faunes de mammifères du Miocène Moyen et Supérieur de la region Lyonnaise. Paleobiologie Continentale, 14 (2): 339-346.

Meulen, A.J. van der \& Bruijn, H. de (1982). The mammals from the Lower Miocene of Aliveri (Island of Evia, Greece). Part 2. The Gliridae. Proceedings Koninklijke Nederlandse Akademie van Wetenschappen, Series B. 85 (4): 485-524.

Meulen, A.J. van der \& Daams, R. (1992). Evolution of Early-Middle Miocenerodent faunas in relation to longterm palaeoenvironmental changes. Palaeogeography, Palaeoclimatology, Palaeoecology, 93: 227-253. http://dx.doi.org/10.1016/0031-0182(92)90099-Q.

Meulen, A.J. van der; Peláez-Campomanes, P. \& Daams, R. (2003). Revision of medium-sized Cricetidae from the Miocene of the Daroca-Villafeliche area in The Calatayud-Teruel basin (Zaragoza, Spain). Coloquios de Paleontología, Vol. Ext.: 385-441.

Morales, J.; Capitán, J.; Calvo, J.P. \& Sesé, C. (1992). Nuevo yacimiento de vertebrados del Mioceno
Superior del Sur de Madrid (Cerro Batallones, Torrejón de Velasco). Geogaceta, 12: 77-80.

Morales, J.; Nieto, M.; Amezua, L.; Fraile, S.; Herráez, E.; Peláez-Campomanes, P.; Salesa, M.J.; Sánchez, I.M. \& Soria, D. (eds.) (2000a). Patrimonio Paleontológico de la Comunidad de Madrid. Arqueología, Paleontología y Etnografía, 6: 371 pp.

Morales, J.; Alcalá, L.; Amezua, L.; Antón, M.; Fraile, S.; Gómez, E.; Montoya, P.; Nieto, M.; Pérez, B.; Salesa, M.J. \& Sánchez, I.M. (2000b). El yacimiento de El Cerro de los Batallones. In: Patrimonio Paleontológico de la Comunidad de Madrid (Morales, J.; Nieto, M.; Amezua, L.; Fraile, S.; Herráez, E.; Peláez-Campomanes, P.; Salesa, M.J.; Sánchez, I.M. \& Soria, D., eds.). Arqueología, Paleontología y Etnografía, 6: 179-190.

Morales, J.; Alcalá, L.; Álvarez Sierra, M.A.; Antón, M.; Azanza, B.; Calvo, J.P.; Carrasco, P.; Fraile, S.; GarcíaParedes, I.; Gómez, E.; Hernández-Fernández, M.; Merino, L.; Meulen, A. van der; Martin Escorza, C.; Montoya, P.; Nieto, M.; Peigné, S.; Pérez, B.; PeláezCampomanes, P.; Pozo, M.; Quiralte, V.; Salesa, M.J.; Sánchez, I.M.; Sánchez Marco, A.; Silva, P.G.; Soria, M.D. \& Turner, A. (2004). Paleontología del sistema de yacimientos de mamíferos miocenos del Cerro de los Batallones, Cuenca de Madrid. Geogaceta, 35: 139-142.

Morales, J.; Pozo. M.; Silva, P.G.; Domingo, M.S.; López-Antoñanzas, R.; Álvarez Sierra, M.A.; Antón, M.; Martín Escorza, C.; Quiralte, V.; Salesa, M.J.; Sánchez, I.M.; Azanza, B.; Calvo, J.P.; Carrasco, P.; García -Paredes, I.; Knoll, F.; Hernández Fernández, M.; Hoek Ostende, L. vanden; Merino, K.; Meulen,A.J.van der; Montoya, P.; Peigné,S.; Peláez-Campomanes, P.; Sánchez-Marco, A.; Turner, A.; Abella, J.; Alcalde, G.M.; Andrés, M.; Miguel, D. de; Cantalapiedra, J.L.; Fraile, S.; García Yelo, B.A.; Gómez Cano, A.R.; López Guerrero, P.; Oliver Pérez, A. \& Siliceo, G. (2008). El sistema de yacimientos de mamíferos miocenos del Cerro de los Batallones, Cuenca de Madrid: estado actual y perspectivas. Palaeontologica Nova, SEPAZ, 8: 41-117.

Peláez-Campomanes, P.;Azanza, B.;Calvo,J.P.; Daams, R.; Herráez, E.; Morales, J.; Nieto,M. \& Soria, D. (2000). Bioestratigrafía de las faunas de mamíferos del Mioceno de Madrid: Datación de las unidades estratigráficas. In: Patrimonio Paleontológico de la Comunidad de Madrid (Morales, J.; Nieto, M.; Amezua, L.; Fraile, S.; Herráez, E.; PeláezCampomanes, P.; Salesa, M.J.; Sánchez, I.M. \& Soria, D., eds.). Arqueología,Paleontología y Etnografía, 6: 103-140.

Peláez-Campomanes, P.; Morales, J.; Álvarez Sierra, M.A.; Azanza, B.; Fraile, S.; García Paredes, I.; Hernández Fernández, M.; Herráez, E.; Nieto, M.; Pérez, B.; Quiralte, V.; Salesa, M.; Sánchez, I.M. \& Soria, D. 
(2003). Updated biochronology of the Miocene mammal faunas from the Madrid basin (Spain). In: Distribution and migration of Tertiary Mammals in Eurasia. Volume in honour of Hans de Bruijn (Reumer, J.W.F. \& Wessels, W., eds.). Deinsea, 10: 431-441.

Perales, R.; Serrano, H.; García Yelo, B.A. \& Hernández Fernández, M. (2009). Inferencias paleoambientales del Mioceno Medio de Somosaguas (Pozuelo de Alarcón, Madrid) basadas en la estructura de tamaños corporales de su fauna de mamíferos. Paleolusitana, 1:317-325.

Portero García. J. M, R. \& Pérez González, A. (Redactores). (1990). Mapa geológico de España. 1/50 000, $2^{\circ}$ serie, Hoja número 558, Majadahonda, IGME (ITGE).

Sesé, C. (1980). Mamíferos del Mioceno Medio de Escobosa de Calatañazor (Soria). Tesis Doctoral. Universidad Complutense de Madrid, 388 pp.

Sesé, C. (2006). Los roedores y lagomorfos del Neógeno de España. Estudios Geológicos, 62 (1): 429-480. http://dx.doi.org/10.3989/egeol.0662138.

Sesé, C.; López Martínez, N. \& Herráez, E. (1985). Micromamíferos (Insectívoros, Roedores y Lagomorfos) de la provincia de Madrid. In: Geología y Paleontología del Terciario Continental de la Provincia de Madrid (Alberdi, M.T., ed.). CSIC-MNCN, Madrid, 29-39.

Soria, D; Amezua, L.; Daams, R.; Fraile, S.; Herráez, E.; Morales, J.; Nieto, M.; Peláez-Campomanes, P.; Salesa, M.J. \& Sánchez, I.M. (2000). Faunas del Mioceno. In: Patrimonio Paleontológico de la Comunidad de Madrid (Morales, J.; Nieto, M.; Amezua, L.; Fraile, S.; Herráez, E.; Peláez-Campomanes, P.; Salesa, M.J.; Sánchez, I.M. \& Soria, D., eds.). Arqueología, Paleontología y Etnografía, 6: 110-129.

Torroba, J.; Gómez de la Peña, L.; Gómez Cano, A.R.; López-Guerrero, P. \& Hernández Fernández, M. (2010). Estudio de los patrones de mortandad de Democricetodon larteti (Schaub 1925) en el yacimiento mioceno de Somosaguas Sur (Pozuelo de Alarcón, Madrid). Cidaris, 30: 325-328.

Vegas, R.; Pérez González, A. \& Míquez, F. (1975). Mapa geológico de España. 1/50 000, $2^{\circ}$ serie, Hoja número 19-23, Getafe, IGME (ITGE).

Weerd, A. van de \& Daams, R. (1978). Quantitative composition of rodent faunas in the Spanish Neogene and paleoecological implications. (I) and (II). Proceedings Koninklijke Nederlandse Akademie van Wetenschappen, Series B. 81 (4): 449-473. 